\title{
Safety and target engagement of an oral small-molecule sequestrant in adolescents with autism spectrum disorder: an open-label phase $1 b / 2 a$ trial
}

\author{
A. Stewart Campbell ${ }^{1,8} \bowtie$, Brittany D. Needham ${ }^{2,8}$, Christopher R. Meyer,8, Joanna Tan', \\ Mary Conrad1, Gregory M. Preston', Federico Bolognani', Srinivas G. Rao', Helen Heussler ${ }^{3}$, \\ Rebecca Griffith ${ }^{4}$, Adam J. Guastella5, Amy C. Janes, ${ }^{6}$, Blaise Frederick ${ }^{7}$, David H. Donabedian ${ }^{1}$ and \\ Sarkis K. Mazmanian $\mathbb{1}^{2 \bowtie}$
}

\begin{abstract}
Autism spectrum disorder (ASD) is defined by hallmark behaviors involving reduced communication and social interaction as well as repetitive activities and restricted interests. ASD represents a broad spectrum, from minimally affected individuals to those requiring intense support, with additional manifestations often including anxiety, irritability/aggression and altered sensory processing. Gastrointestinal (GI) issues are also common in ASD, and studies have identified changes in the gut microbiome of individuals with ASD compared to control populations, complementing recent findings of differences in gut-derived metabolites in feces and circulation. However, a role for the GI tract or microbiome in ASD remains controversial. Here we report that an oral GI-restricted adsorbent (AB-2004) that has affinity for small aromatic or phenolic molecules relieves anxiety-like behaviors that are driven by a gut microbial metabolite in mice. Accordingly, a pilot human study was designed and completed to evaluate the safety of AB-2004 in an open-label, single-cohort, multiple-ascending-dose clinical trial that enrolled 30 adolescents with ASD and GI symptoms in New Zealand and Australia. AB-2004 was shown to have good safety and tolerability across all dose levels, and no drug-related serious adverse events were identified. Significant reductions in specific urinary and plasma levels of gut bacterial metabolites were observed between baseline and end of AB-2004 treatment, demonstrating likely target engagement. Furthermore, we observed improvements in multiple exploratory behavioral endpoints, most significantly in post hoc analysis of anxiety and irritability, as well as $\mathrm{GI}$ health, after $\mathbf{8}$ weeks of treatment. These results from an open-label study (trial registration no. ACTRN12618001956291) suggest that targeting gut-derived metabolites with an oral adsorbent is a safe and well-tolerated approach to improving symptoms associated with ASD, thereby emboldening larger placebo-controlled trials.
\end{abstract}

$\mathrm{C}$ urrent estimates of ASD prevalence reach one in 54 children born in the United States ${ }^{1}$, and recent clinical failures $^{2-9}$ highlight the need to expand drug development efforts. Behavioral features and severity are measured by validated observational assessment tools, as there are no imaging-based or molecular biomarkers that reliably and objectively diagnose ASD. Furthermore, autism manifests across a broad spectrum, from minimally affected individuals to those who require intense support, and there are no approved drugs for core symptoms. The etiology of ASD is poorly understood and likely multifactorial but is known to involve complex genetic risks ${ }^{10}$, with over 100 genes implicated to date, each with a small effect size ${ }^{11}$. A role for environmental risks in ASD has also been proposed, encompassing diet ${ }^{12}$, maternal infection $^{13}$, exposure to toxins ${ }^{14}$ and changes in the gut microbiome ${ }^{15}$. The notion that fixed genetic predispositions coupled with variable environmental risks together manifest symptom severity is intriguing from a therapeutic perspective, because correcting mutations in the genome remains challenging, and reducing potential environmental contributors is likely more tractable. Recent studies suggest that molecules produced in the GI tract can enter systemic circulation and affect immunity ${ }^{16}$, metabolism ${ }^{17}$ and behavior ${ }^{18}$. Altered immune and metabolic profiles have been associated with various neuropsychiatric disorders, such as $\mathrm{ASD}^{19-21}$, and the microbiome and metabolome are altered in individuals with $\mathrm{ASD}^{19,22}$, anxiety ${ }^{23}$, depression ${ }^{24}$ and schizophrenia ${ }^{25}$. Notably, over a dozen studies have shown changes in the fecal microbiome in several ASD cohorts compared to controls ${ }^{26}$, although these associations do not resolve cause or effect. Dietary habits likely contribute to the ASD microbiome $^{27}$. Several studies have also revealed changes in the metabolome of individuals with ASD from diverse geographies ${ }^{19,28-30}$, with reports showing dysregulation of the fecal metabolome ${ }^{19,31-33}$. We previously identified several gut microbial metabolites that correlate with ASD-like symptoms in mice, and administration of one of these metabolites, 4-ethylphenyl sulfate (4EPS), to naive animals

'Axial Therapeutics, Woburn, MA, USA. ${ }^{2}$ Division of Biology and Biological Engineering, California Institute of Technology, Pasadena, CA, USA. ${ }^{3}$ Centre for Clinical Trials in Rare Neurodevelopmental Disorders, Queensland Children's Hospital, Brisbane, QLD, Australia. ${ }^{4}$ Optimal Clinical Trials, Auckland, New Zealand. ${ }^{5}$ Brain and Mind Centre, Children's Hospital Westmead Clinical School, Faculty of Medicine and Health, University of Sydney, Sydney, NSW, Australia. ${ }^{6}$ Consulting Neuroscientist to Axial Therapeutics, Woburn, MA, USA. ${ }^{7}$ Consulting MR Physicist to Axial Therapeutics, Woburn, MA, USA.

${ }^{8}$ These authors contributed equally: A. Stewart Campbell, Brittany D. Needham, Christopher R. Meyer. $\bowtie$ e-mail: stewart@axialtx.com; sarkis@caltech.edu 
$\mathbf{a}$

\begin{tabular}{|c|c|}
\hline \multicolumn{2}{|c|}{ Safety population $(n=30)$} \\
\hline Age (years) & $14.2 \pm 1.4$ \\
\hline Race/ethnicity & $>70 \%$ non-Hispanic or Latino $(22 / 30)$ \\
\hline Weight & $57.61 \pm 12.97 \mathrm{~kg}$ \\
\hline BMI & $21.2 \pm 5.31 \mathrm{~kg} \mathrm{~m}^{-2}$ \\
\hline 6-GSI & $3.3 \pm 2.5$ \\
\hline ADOS severity (at screening) & \\
\hline Mild & $1(3.3 \%)$ \\
\hline Moderate & $9(30 \%)$ \\
\hline Severe & $20(66.6 \%)$ \\
\hline \multicolumn{2}{|c|}{ Secondary endpoints } \\
\hline \multicolumn{2}{|l|}{ Clinical questionnaires } \\
\hline CGI-S & $3.5 \pm 0.9(n=26)$ \\
\hline PARS & $10.5 \pm 4.5(n=26)$ \\
\hline \multicolumn{2}{|l|}{ Subject/parent-caregiver questionnaires } \\
\hline BSS & $3.7 \pm 1.0(n=26)$ \\
\hline NRS & $1.7 \pm 1.7(n=26)$ \\
\hline GSRS & $1.9 \pm 1.2(n=26)$ \\
\hline ABC-I & $12.2 \pm 11.0(n=26)$ \\
\hline ABC-SW & $13.9 \pm 9.9(n=26)$ \\
\hline SRS-2 & $76.7 \pm 11.3(n=26)$ \\
\hline RBS-R & $45.4 \pm 33.4(n=25)$ \\
\hline VABS & $71.6 \pm 12.6(n=15)$ \\
\hline
\end{tabular}

b

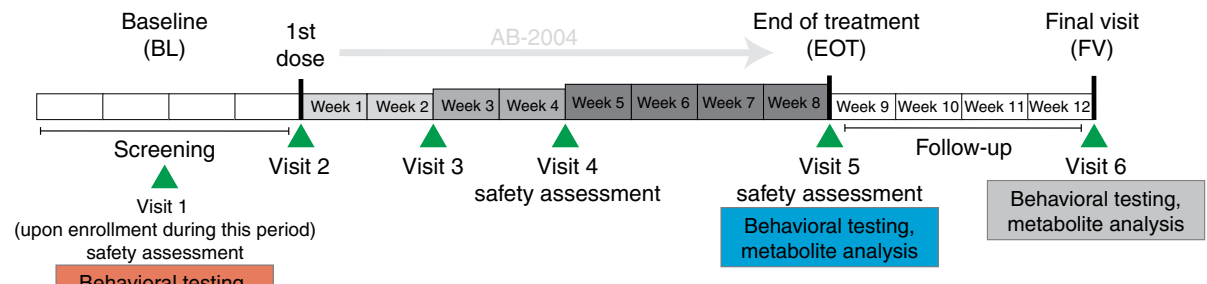

Fig. 1 | Demographics of clinical trial participants and trial schedule. a, Trial demographics and metadata summary of participants. b, Phase 1 clinical trial schedule. Participants were screened during a 4-week run-in period, followed by dose escalation in weeks 0-2, weeks 2-4 and weeks 4-8, with a follow-up 4 weeks after trial. ABC-SW, Aberrant Behavior Checklist-Social Withdrawal; BMI, body mass index.

induced an anxiety-like phenotype ${ }^{34}$. 4EPS and several structurally related molecules are dysregulated in the feces and plasma of individuals with ASD compared to control populations ${ }^{19}$. Furthermore, an open-label fecal transplant study described improvements in GI and behavioral parameters in 18 individuals with $\mathrm{ASD}^{35,36}$, with a partial restoration of metabolomic profiles ${ }^{37}$. Although these early studies remain speculative, the prospect for ASD interventions targeting the microbiome or metabolome are both conceptually attractive and practically realizable.

AB-2004, known otherwise as AST-120 (ref. ${ }^{38}$ ), is a highsurface-area spherical carbon adsorbent that has affinity for uremic toxins, including those of gut bacterial origin, such as the simple phenols, 4EPS, $p$-cresol sulfate (pCS) and $p$-cresol glucuronide (pCG), as well as the indole derivative 3-indoxyl sulfate (3IS) and hippuric acid ${ }^{39}$, based on evidence from rodent models and patients with chronic kidney disease ${ }^{40}$ and irritable bowel syndrome ${ }^{41}$. Taken orally, it binds and sequesters related aromatic metabolites as it passes through the GI tract without being absorbed and is ultimately excreted, effectively lowering systemic metabolite exposure. We hypothesized that AB-2004 would also reduce the structurally related 3-hydroxyhippurate (HHA) and phenylpropanoic acids or other related small-molecule metabolites, such as 3-(3-hydroxyphenyl)-3-hydroxypropionate (HPHPA), 3-(4-hydroxyphenyl)propionate (HPPA), 3-hydroxyphenylacetate (HPAA), 3-carboxy-4-methyl-5-propyl-2-furanpropanoate (CMPF) and imidazolepropionate (IPA). There is accumulating evidence that increased levels of this chemical class of microbial metabolites is associated with $\mathrm{ASD}^{37,42,43}$. For instance, 4EPS, pCS, 3IS, hippuric acid and hydroxyphenylacetic acid metabolite levels have been found to be elevated in children with $\mathrm{ASD}^{19,20,28,42,44-47}$, and levels of some of these metabolites also correlate with GI and behavioral symptoms ${ }^{19,20,42,44}$. Unpublished preclinical work suggests that these findings might go beyond simple associations between microbial metabolites and behavioral endpoints; namely, production of 4EPS by gut bacteria results in changes in brain cell function and increases anxiety-like and ASD-like behaviors in mice (see accompanying manuscript, accepted for publication). pCS administered to mice leads to deficits in social communication and repetitive behaviors ${ }^{48}$, and both pCS and 3IS promote anxiety-like and depression-like features in rodents ${ }^{49-51}$. To date, no studies have attempted to modify the production or concentrations of this class of compound in human neuropsychiatric disorders.

\section{Results}

AB-2004 reduces 4EPS and anxiety-like behavior in mice. We recently reported that 4 EPS is elevated in the plasma of individuals with $\mathrm{ASD}^{34}$, although bacterial sources for production of the metabolite remained unknown. The gut microbiome is predicted to harbor genes that convert tyrosine, the precursor of several mammalian neurotransmitters, to 4-ethylphenol (4EP), which could then be 
sulfated to 4EPS. Sulfation in the liver or other organs is a common detoxifying activity in mice and humans for structurally related phenolic molecules ${ }^{52}$. We systematically tested several bacterial species for the enzymatic activity required for biosynthesis of $4 \mathrm{EP}$ from tyrosine and then cloned genes that showed predicted activity into genetically tractable strains of gut bacteria. Subsequently, gnotobiotic mice were colonized with isogenic bacterial strains that

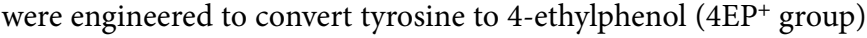
or mutants of the same strains that lack genes encoding enzymes

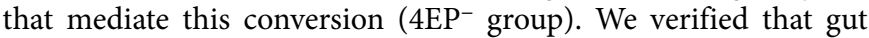
microbial production of $4 \mathrm{EP}$, followed by efficient host sulfation, leads to the presence of $4 \mathrm{EPS}$ in urine of $4 \mathrm{EP}^{+}$mice (Extended Data Fig. 1a-c; control). 4EPS promoted anxiety-like behavior in several testing paradigms: (1) open-field exploration where mice ventured less into the more exposed zone of the arena; (2) the elevated plus maze (EPM) where $4 \mathrm{EP}^{+}$mice spent less time in the terminus of the open arms; and (3) the marble burying test (Extended Data Fig. 1f-i). (For further details, see Methods and the accompanying manuscript). This experimental paradigm enables effective testing of drugs that neutralize 4EPS.

After colonization, both groups of mice were placed on a diet consisting of $5 \% \mathrm{AB}-2004$ by weight of chow or matched control diet 2 weeks before behavior testing (Extended Data Fig. 1b). As expected, systemic levels of 4EPS measured in urine were lowered by oral AB-2004 administration (Extended Data Fig. 1c). There were no differences in bacterial colonization levels; weight gain was not significantly influenced (Extended Data Fig. 1d,e); and no signs of distress, illness or other differences were observed among the mouse groups. Behavioral analysis revealed that treatment with AB-2004 ameliorated behavioral deficits in the open field and EPM tests for anxiety (Extended Data Fig. 1f-h). Specifically, 4EP mice given AB-2004 spent more time exploring the exposed (that is, riskier) areas of the tests compared to $4 \mathrm{EP}^{+}$mice on the control diet. AB-2004 treatment also stabilized performance in the anxiety/ repetitive behavior-related marble burying test (Extended Data Fig. 1i). These results were accompanied by trending improvements in the grooming test for repetitive behavior (Extended Data Fig. 1j). Results from this simplified mouse model indicate that AB-2004 was effective in reducing systemic levels of 4EPS and preventing 4EPS-induced anxiety-like behaviors. These preclinical findings, coupled with the known safety profile of AB-2004, provided justification for a human trial.

Phase 1 clinical trial design. We designed and conducted an open-label, phase $1 \mathrm{~b} / 2 \mathrm{a}$ clinical trial at three sites in New Zealand and Australia with primary endpoints for safety and tolerability as determined by reported and observed adverse effects and laboratory results. Secondary endpoints included target engagement, which was assessed objectively by measuring microbially derived metabolites in plasma and urine. Behavioral endpoints were exploratory. At screening, ASD diagnosis was confirmed using the Autism Diagnostic Observational Schedule, 2nd Edition (ADOS-2), and the presence of GI symptoms was verified through the Gastrointestinal Severity Index (6-GSI) ${ }^{53}$ and a $14-d$ bowel habit diary. Thirty adolescents (29 males and one female) previously diagnosed with ASD (Fig. 1a and Extended Data Fig. 2a) met the inclusion criteria as outlined in Extended Data Table 1. After enrollment, each participant was administered baseline behavioral assessments using the Pediatric Anxiety Rating Scale (PARS) ${ }^{54}$, the Aberrant Behavior Checklist $(\mathrm{ABC})^{55}$, the Social Responsiveness Scale (SRS-2) ${ }^{56}$, the Repetitive Behavior Scale Revised (RBS-R) ${ }^{57}$ and the Vineland Adaptive Behavior Score (VABS-3) ${ }^{58}$, and several gastrointestinal symptom metrics were measured, including the $6-\mathrm{GSI}^{53}$, the Bristol Stool Scale (BSS) ${ }^{59}$ and the Gastrointestinal Symptom Rating Scale (GSRS) ${ }^{60}$. All inclusion and exclusion criteria can be found in Extended Data Table 1. Study participants were asked to take
Table 1 | Treatment-emergent adverse events

\begin{tabular}{ll}
$\begin{array}{l}\text { Adverse event system organ } \\
\text { class/preferred term }\end{array}$ & $\begin{array}{l}\text { Safety population Severity } \\
(n=30)\end{array}$ \\
\cline { 2 - 2 } & $n(\%) \mathrm{E}$
\end{tabular}

Total

Overall study $24(80.0 \%) 68 \quad \begin{aligned} & \text { Mild, Moderate, } \\ & \text { Severe }\end{aligned}$

Related

$\begin{array}{lll}\text { Overall study } & 0(0 \%) 0 \quad \mathrm{~N} / \mathrm{A}\end{array}$

Possibly related

Overall study $\quad 14(46.7 \%) 21 \quad$ Mild, Moderate

GI disorders

Abdominal pain

$2(6.7 \%) 2 \quad$ Mild

Nausea

$2(6.7 \%) 2 \quad$ Mild

Diarrhea

$1(3.3 \%) 2$

Mild, Moderate

Abdominal discomfort

$1(3.3 \%) 1$

Mild

Abdominal tenderness

$1(3.3 \%) 1$

Mild

Dyspepsia

$1(3.3 \%) 1$

Mild

Rectal hemorrhage

$1(3.3 \%) 1$

Mild

Abdominal pain upper

$1(3.3 \%) 1$

Moderate

General disorders

Pyrexia

$2(6.7 \%) 2$

Mild

Nervous system disorders

Headache

$3(10.0 \%) 3 \quad$ Mild

Disturbance in attention

$1(3.3 \%) 1$

Mild

Psychiatric disorders

$\begin{array}{lll}\text { Insomnia } & 1(3.3 \%) 1 & \text { Moderate } \\ \text { Anxiety } & 1(3.3 \%) 1 & \text { Moderate }\end{array}$

Respiratory, thoracic and mediastinal disorders

Epistaxis 1(3.3\%) $1 \quad$ Mild

Skin/subcutaneous tissue disorders

$\begin{array}{lll}\text { Rash } & 1(3.3 \%) 1 & \text { Moderate }\end{array}$

Not related

Overall study $21(70.0 \%) 47 \quad$ Mild, Moderate,

Severe

Categories are indicated in bold. \%, percent of participants with events; $E$, number of events; $n$, number of participants with events; N/A, not applicable.

three daily, weight-adjusted (see Methods for details) ascending oral doses of AB-2004 totaling $\leq 2.25 \mathrm{~g}$ per day, escalating to $\leq 4.5 \mathrm{~g}$ per day at 2 weeks and $\leq 6 \mathrm{~g}$ per day daily at 4 weeks until the end of treatment on week 8 , with a final visit 4 weeks after the end of treatment (Fig. 1b). Urine, blood and stool samples were collected, and behavioral assessment performed at baseline (BL), end of treatment (EOT) and final visit (FV), with continuous health monitoring throughout (Fig. 1b and Extended Data Table 2). Twenty-seven of 30 enrolled participants completed to the EOT, but one was removed due to Coronavirus Disease 2019 (COVID-19) schedule interruptions, resulting in a completers group of 26 . Twenty-four participants completed to the FV.

AB-2004 is safe and well tolerated in adolescents with ASD. Assessment of overall health, including GI symptoms, was determined by the Clinical Global Impressions scale for severity and improvement (CGI-S and CGI-I, respectively). From BL to EOT, 
a

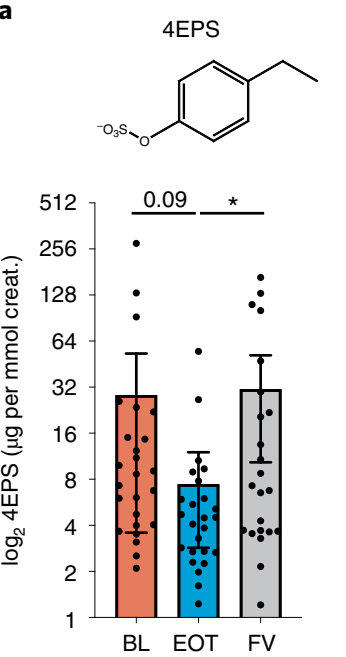

b<smiles>CCc1ccc(C)cc1</smiles>

c<smiles>C1CCC1</smiles><smiles>CCc1ccc(C)cc1</smiles>

d

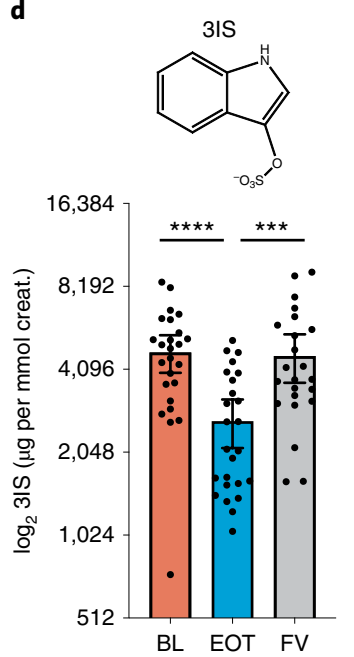

e<smiles>O=C(O)Cc1ccc(O)cc1</smiles><smiles>O=C(O)CC(c1ccccc1)c1ccc(Cl)cc1</smiles>

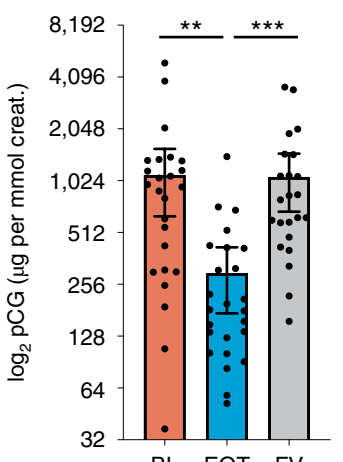

BL EOT FV

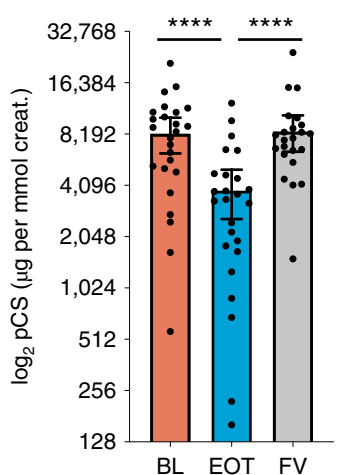

BL EOT FV

g<smiles>O=C(O)CNC(=O)c1cccc(O)c1</smiles>

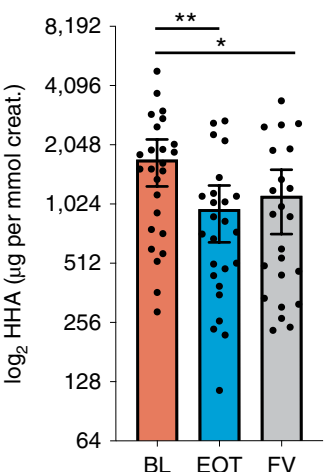

Fig. 2 | Oral AB-2004 treatment reduces levels of several bacterial metabolites. a-f, Metabolite levels in urine from BL (orange), EOT (blue) and FV (gray) time points from all participants, normalized to creatinine ( $\mu$ g metabolite per mmol creatinine) $\operatorname{on} \log _{2}$ scale. Exact adjusted $P$ values: 4 EPS, 0.03; pCG, 0.002, 0.0003; pCS, <0.0001, <0.0001; 3IS, <0.0001, 0.0003; HPAA, 0.002; HPHPA, 0.0095; and HHA, 0.005, 0.02. Chemical structures are shown above associated data panels. Urine from one participant could not be obtained due to incontinence. Data analysis was conducted on the completers group ( $n=26$, with two missing data points in the FV time point because two participants missed assessment due to parental illness). Analysis is exploratory and post hoc in nature, shown as mean and $95 \%$ confidence interval analyzed with a linear mixed-effects model with Geisser-Greenhouse correction, multiple comparisons and false discovery rate correction across all metabolites (nominal $P$ values: ${ }^{\star} P_{\text {adj }} \leq 0.05 ;{ }^{* \star} P_{\text {adj }} \leq 0.01$; ${ }^{\star \star \star} P_{\text {adj }} \leq 0.001$; $\left.{ }^{\star \star \star \star} P_{\text {adj }} \leq 0.0001\right)$. creat., creatinine.

$76.9 \%$ of participants (20 of 26) improved at least one point on the CGI-I scale (Extended Data Fig. 2b). Although GI symptoms were an inclusion criterion based on 6-GSI and a 14-d bowel habit e-diary assessment during screening, $19.2 \%$ of participants presented with no clinical GI disorder based on the CGI at the time of assessment (including normal and borderline scores). Notably, the number of participants with no measurable GI symptoms doubled from BL to EOT (19.2\% to 38.5\%) (Extended Data Fig. 2c).

Median adherence to dosing was $97.5 \%$, and no laboratory concerns arose, showing that AB-2004 was well tolerated. Notably, overall safety metrics showed that no serious adverse events related to the drug or any deaths occurred during the reporting period of the study. Most mild or moderate adverse effects were in the GI category, including abdominal pain and nausea (Table 1). The study, therefore, met its primary endpoints for safety and tolerability, extending the safety record of this drug to an adolescent ASD population for the first time.
Microbial metabolite levels are lowered by AB-2004. Based on the known pharmacology of binding phenolic compounds and its practically complete lack of systemic absorption, we hypothesized that oral AB-2004 would diminish levels of specific gut-derived metabolites in circulation by facilitating their excretion in the feces. As predicted, AB-2004 treatment resulted in reduced levels of 4EPS, pCG, pCS, 3IS, HPHPA and HPAA in urine from the BL to EOT time points (Fig. 2 and Extended Data Fig. 3a), with similar profiles in plasma (Extended Data Fig. 3b). Concentrations between urine and plasma were highly correlated for many metabolites (Extended Data Fig. 3c). Urine metabolites largely rebounded to pre-drug (BL) levels at the FV time point 4 weeks after treatment had concluded, supporting the conclusion that metabolite levels were influenced directly by AB-2004 administration (Fig. 2). N-acetyl serine levels, which were measured as a control metabolite not bound by AB-2004, did not change in urine or plasma between BL and EOT (Extended Data Fig. 3d). These data indicate that target engagement 
of gut-derived microbial metabolites by AB-2004 can effectively reduce their systemic levels.

Oral AB-2004 might alter brain connectivity. As a preliminary look into potential brain activity patterns, we performed resting state functional magnetic resonance imaging (fMRI) on a small subset of ten study participants to estimate connectivity between brain regions. Two 5-min scans were conducted at BL and EOT time points focusing on changes in regions associated with emotional behavior responses. This included regions such as the amygdala, which is crucial for emotional processing networks such as those involving anxiety ${ }^{61}$, and the anterior cingulate cortex (ACC), which is involved in emotional and cognitive networks ${ }^{62}$. Atypical activity in one or both of these regions has been observed in preclinical studies $^{63}$ and in ASD cohorts ${ }^{64-68}$. We observed a decrease in coupling between the amygdala and the rostral anterior cingulate cortex (rACC) (Extended Data Fig. 4a), an encouraging outcome because higher amygdala-rACC connectivity is associated with higher anxiety ${ }^{69}$. This pilot finding suggests that further study of functional connectivity in the amygdala and ACC might provide insights into mechanisms of action of AB-2004 and the metabolites it binds.

Exploratory outcomes show improvement of core ASD behavior. In addition, we captured several domains of behavioral data for all study participants as exploratory endpoints. The $\mathrm{VABS}^{58}$ was administered at BL, EOT and FV, and overall scores, as well as communication and socialization scores, were significantly increased by EOT (Extended Data Fig. 4b-e). Although ten participants were removed from this analysis owing to incomplete data, thereby limiting the sample size of this comparison, a mean increase of 7.8 points from BL to EOT in the Adaptive Behavior Composite score is above the minimal clinically relevant cutoff of 3.75 points $^{70,71}$ (Extended Data Fig. 4b). Modest indications of improved social communication and repetitive behavior of study participants was also detected in other behavioral assessments, including the SRS (Extended Data Fig. 5a-f) and, to a lesser extent, in the ABC (Extended Data Fig. 5g-j). Importantly, for our study, both assessment tools have been used to evaluate the efficacy of ASD therapeutics ${ }^{72-74}$. Future studies will aim to confirm the encouraging, but preliminary, improvements observed here in social communication and repetitive behavior, as these outcome measures are likely to be influenced by placebo effects ${ }^{75}$.

AB-2004 reduced anxiety and irritability. We report the most striking behavioral outcomes of AB-2004 treatment in two highly prevalent non-core domains of ASD, namely anxiety and irritability. In particular, study participants with elevated BL anxiety scores $\geq 10$, as measured by the PARS test, showed marked improvements in anxiety between initial (BL) and last (EOT) dose, a positive effect that persisted 1 month after withdrawal of drug (FV) (Fig. 3a,b). These results indicate that AB-2004 might be effective in treating elevated anxiety in individuals with ASD, as the minimum clinically important change in the PARS score is $15 \%{ }^{76}$, and our results show an average improvement of $30 \%$ in the group with anxiety at $\mathrm{BL}$, with nine of 15 individuals with elevated anxiety, resulting in a diagnostic score that qualified as remission (score of 10 or lower).

Irritability is also frequent in the ASD population and can be assessed as part of the ABC scale ${ }^{55}$. We observed a significant overall decrease in irritability as measured by the ABC-I subscale between $\mathrm{BL}$ and EOT (Fig. 3c). In particular, participants with high BL irritability (scores $\geq 15$ ), which represents the top quartile of severity within the ASD population as a whole ${ }^{77}$, displayed a remarkable 9.1-point decrease in ABC-I (Fig. 3d). The improvements at EOT were largely mitigated after drug washout $(\mathrm{FV})$ in most participants (Fig. 3d). Correlations between a single metabolite and behavioral scores were not clear (Extended Data Table 3), and analysis to understand the potential effect of multiple metabolite interactions

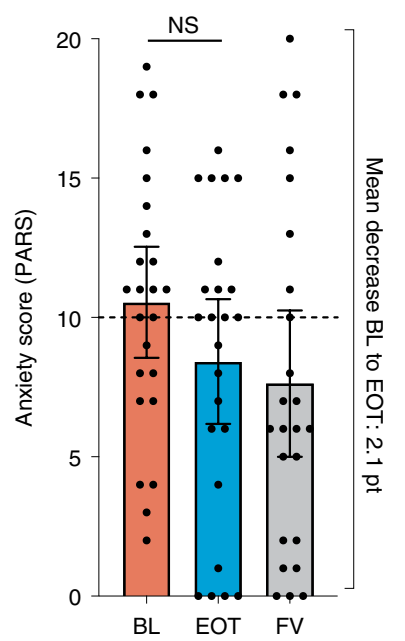

c

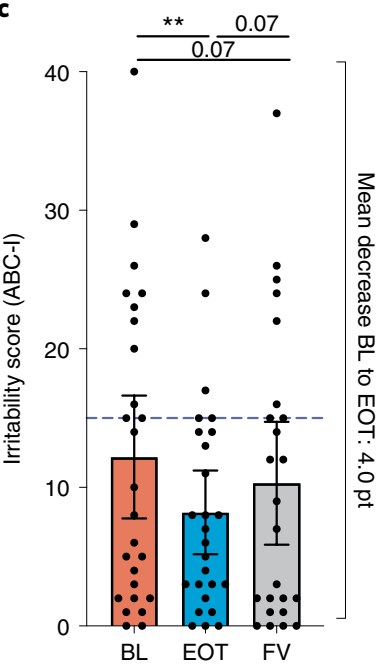

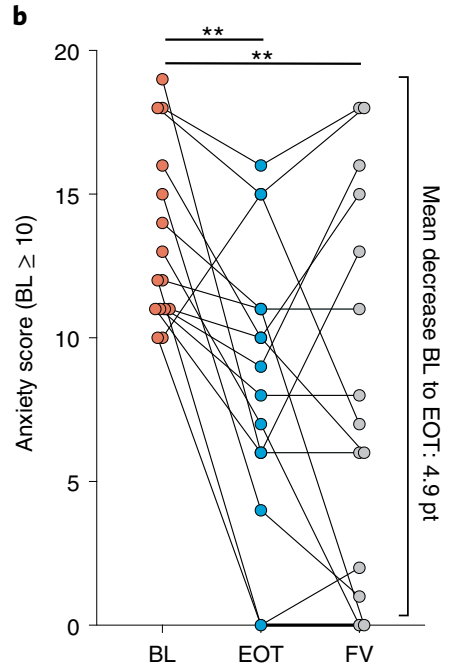

d

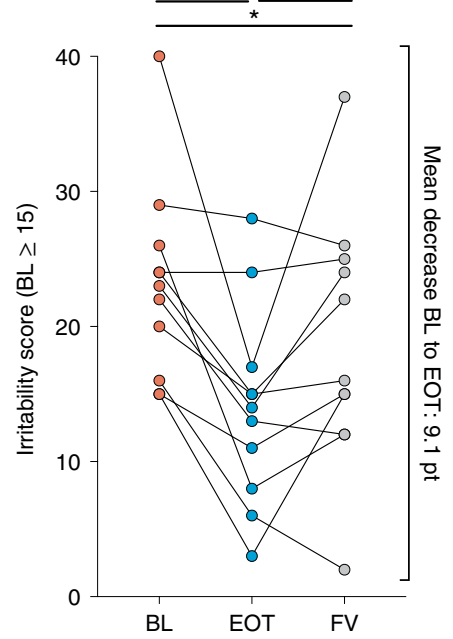

Fig. 3 | AB-2004 administration improves anxiety and irritability, especially in individuals with high BL scores. a, Anxiety (measured by PARS) scores of all eligible study participants at BL (orange), EOT (blue) and FV (gray) time points, with mean test scores represented as bars. Dotted line indicates threshold for anxiety $(n=24)$. b, PARS anxiety scores of the subset of participants scoring $\geq 10$ at $B \mathrm{~L}$, showing participant change to EOT and FV $(n=15)$. Exact adjusted $P$ values: BL versus EOT, $0.001 ; B L$ versus FV, 0.001. c, Irritability (measured by ABC-I) scores of all eligible study participants at BL, EOT and FV time points. Dotted line indicates threshold for the top quartile of irritability severity among the ASD population (BL and EOT, $n=26 ; \mathrm{FV}, n=24$ owing to a missing FV value for two participants). Exact adjusted $P$ value: 0.008 . d, Irritability (ABC-I) scores of the subset of participants scoring $\geq 15$ at $B \mathrm{~L}$, showing participant change to EOT and $\mathrm{FV}(n=11)$. Exact adjusted $P$ values: $\mathrm{BL}$ versus EOT, 0.003; EOT versus FV, $0.04 ; \mathrm{BL}$ versus $\mathrm{FV}, 0.04$. Data analysis was conducted on the completers group ( $n=26$, with two missing data points at the FV time point in $A B C-I$ and at all time points in PARS because two participants missed assessments due to parental illness). Analysis is exploratory and post hoc in nature, shown as mean and $95 \%$ confidence interval. Analyses were performed by repeated-measures one-way ANOVA ( $\mathbf{a}, \mathbf{b}$ and $\mathbf{d}$ ) or the linear mixed-effects model (c) with Geisser-Greenhouse correction, multiple comparisons and false discovery rate correction within each test (nominal $P$ values: $\left.{ }^{\star} P_{\text {adj }} \leq 0.05 ;{ }^{\star \star} P_{\text {adj }} \leq 0.01\right)$. NS, not significant.; pt, points.

is underway. These data reveal that almost all study participants with elevated anxiety or irritability showed significant behavioral improvements after 8 weeks of treatment with AB-2004. 


\section{Discussion}

Based on data from the recently completed open-label trial described here, AB-2004 is safe and well tolerated for use in an adolescent ASD population, with no serious adverse events related to the drug. Our studies also suggest target engagement by AB-2004, as evidenced by reduced levels of gut microbial metabolites in plasma and urine after 8 weeks of treatment and a general rebound to BL levels after 4 weeks of drug washout. Furthermore, AB-2004 decreased the number of participants presenting with GI symptoms; however, it is unclear whether intestinal issues are linked to other endpoints. Although our study was powered for safety and tolerability, we surprisingly observed indicators of improvements in ASD-associated behaviors, namely anxiety and irritability. Decreased anxiety persisted after drug removal, whereas improvements in irritability largely returned to $\mathrm{BL}$ levels by the FV. We cannot exclude a contribution for metabolites bound by AB-2004 that were not measured here, of host, dietary or microbial origin. Also, our study does not resolve indirect effects of drug through potential changes in nutrition, immune status and GI function, for example, and further proof of mechanism will require additional work. However, to our knowledge, this is the first interventional study linking phenolic metabolites in the gut with clinical features of ASD. Although the preliminary evidence for improvements in behavior within this small ASD cohort are encouraging, the absence of a control arm necessitates double-blinded, placebo-controlled trials to confirm the efficacy of AB-2004.

There are currently no approved pharmacological therapies for the treatment of the core symptoms of $\mathrm{ASD}^{78}$. Two drugs, risperidone and aripiprazole, are currently approved by the US Food and Drug Administration (FDA) for treatment of irritability in individuals with $\mathrm{ASD}^{79}$. Irritability behaviors are common in pediatric ASD and have major implications in child development, receptivity to behavioral therapy and child/caregiver health-related quality of life ${ }^{80}$. Both drugs are atypical anti-psychotic medications and are associated with a range of side effects ${ }^{7,81}$, such as somnolence, metabolic changes, weight gain, leukopenia and tardive dyskinesia ${ }^{73,82-84}$. In a phase 3 study of aripiprazole ${ }^{85}$ with inclusion criteria based on high irritability levels $(A B C-I \geq 18)$, the response rate, or percentage of individuals with $25 \%$ improvement in ABC-I scores and a CGI-I $\leq 2$, was $49-56 \%$ in the drug arms, with a $34.7 \%$ response rate in the placebo arm. In our study, a post hoc analysis showed a $75 \%$ and $82 \%$ response rate in subgroups meeting somewhat similar criteria (ABC-I 18 or 15, respectively), providing optimism for future, larger placebo-controlled studies with AB-2004. Importantly, $\mathrm{ABC}$-I is a validated instrument for measuring irritability in individuals with ASD and was used as the primary efficacy measurement in pivotal trials leading to FDA approval of aripiprazole and risperidone; therefore, a regulatory path for the development of AB-2004 in this patient population is already established. The favorable clinical safety profile makes AB-2004 an attractive potential alternative to the currently available drugs for irritability in ASD. Based on findings from this safety and tolerability study, a placebo-controlled randomized trial is now underway to test the effects of AB-2004 in an ASD cohort powered to report changes in irritability.

\section{Online content}

Any methods, additional references, Nature Research reporting summaries, source data, extended data, supplementary information, acknowledgements, peer review information; details of author contributions and competing interests; and statements of data and code availability are available at https://doi.org/10.1038/ s41591-022-01683-9.

Received: 25 June 2021; Accepted: 5 January 2022;

Published online: 14 February 2022

\section{References}

1. Maenner, M. J. et al. Prevalence of autism spectrum disorder among children aged 8 years-autism and developmental disabilities monitoring network, 11 sites, United States, 2016. MMWR Surveill. Summ. 69, 1-12 (2020).

2. Warren, Z. et al. A systematic review of early intensive intervention for autism spectrum disorders. Pediatrics 127, e1303-e1311 (2011).

3. Potter, L. A. et al. A randomized controlled trial of sertraline in young children with autism spectrum disorder. Front. Psychiatry 10, 810 (2019).

4. Krishnaswami, S., McPheeters, M. L. \& Veenstra-Vanderweele, J. A systematic review of secretin for children with autism spectrum disorders. Pediatrics 127, e1322-e1325 (2011).

5. King, B. H. et al. Lack of efficacy of citalopram in children with autism spectrum disorders and high levels of repetitive behavior: citalopram ineffective in children with autism. Arch. Gen. Psychiatry 66, 583-590 (2009).

6. Loebel, A. et al. Lurasidone for the treatment of irritability associated with autistic disorder. J. Autism Dev. Disord. 46, 1153-1163 (2016).

7. Goel, R., Hong, J. S., Findling, R. L. \& Ji, N. Y. An update on pharmacotherapy of autism spectrum disorder in children and adolescents. Int. Rev. Psychiatry 30, 78-95 (2018).

8. Hardan, A. Y. et al. Efficacy and safety of memantine in children with autism spectrum disorder: results from three phase 2 multicenter studies. Autism 23, 2096-2111 (2019).

9. Veenstra-VanderWeele, J. et al. Arbaclofen in children and adolescents with autism spectrum disorder: a randomized, controlled, phase 2 trial. Neuropsychopharmacology 42, 1390-1398 (2017).

10. Rylaarsdam, L. \& Guemez-Gamboa, A. Genetic causes and modifiers of autism spectrum disorder. Front. Cell. Neurosci. 13, 385 (2019).

11. Brueggeman, L., Koomar, T. \& Michaelson, J. J. Forecasting risk gene discovery in autism with machine learning and genome-scale data. Sci. Rep. 10, 4569 (2020)

12. Zhong, C., Tessing, J., Lee, B. K. \& Lyall, K. Maternal dietary factors and the risk of autism spectrum disorders: a systematic review of existing evidence. Autism Res. 13, 1634-1658 (2020).

13. Jiang, H.-Y. et al. Maternal infection during pregnancy and risk of autism spectrum disorders: a systematic review and meta-analysis. Brain Behav. Immun. 58, 165-172 (2016).

14. Rossignol, D. A., Genuis, S. J. \& Frye, R. E. Environmental toxicants and autism spectrum disorders: a systematic review. Transl. Psychiatry 4, e360 (2014).

15. Ho, L. K. H. et al. Gut microbiota changes in children with autism spectrum disorder: a systematic review. Gut Pathog. 12, 6 (2020).

16. Garrett, W. S. Immune recognition of microbial metabolites. Nat. Rev. Immunol. 20, 91-92 (2020).

17. Fan, Y. \& Pedersen, O. Gut microbiota in human metabolic health and disease. Nat. Rev. Microbiol. 19, 55-71 (2021).

18. Needham, B. D., Kaddurah-Daouk, R. \& Mazmanian, S. K. Gut microbial molecules in behavioural and neurodegenerative conditions. Nat. Rev. Neurosci. 21, 717-731 (2020).

19. Needham, B. D. et al. Plasma and fecal metabolite profiles in autism spectrum disorder. Biol. Psychiatry 89, 451-462 (2020).

20. Ming, X., Stein, T. P., Barnes, V., Rhodes, N. \& Guo, L. Metabolic perturbance in autism spectrum disorders: a metabolomics study. J. Proteome Res. 11, 5856-5862 (2012).

21. Adams, J. B., Vargason, T., Kang, D.-W., Krajmalnik-Brown, R. \& Hahn, J Multivariate analysis of plasma metabolites in children with autism spectrum disorder and gastrointestinal symptoms before and after microbiota transfer therapy. Processes 7, 806 (2019).

22. Garcia-Gutierrez, E., Narbad, A. \& Rodríguez, J. M. Autism spectrum disorder associated with gut microbiota at immune, metabolomic, and neuroactive level. Front. Neurosci. 14, 578666 (2020).

23. Humer, E., Pieh, C. \& Probst, T. Metabolomic biomarkers in anxiety disorders. Int. J. Mol. Sci. 21, 4784 (2020).

24. Konjevod, M. et al. Metabolomics analysis of microbiota-gut-brain axis in neurodegenerative and psychiatric diseases. J. Pharm. Biomed. Anal. 194, 113681 (2021)

25. Li, C. et al. Metabolomics in patients with psychosis: a systematic review. Am. J. Med. Genet. B Neuropsychiatr. Genet. 177, 580-588 (2018).

26. Pulikkan, J., Mazumder, A. \& Grace, T. Role of the gut microbiome in autism spectrum disorders. Adv. Exp. Med. Biol. 1118, 253-269 (2019).

27. Yap, C. X. et al. Autism-related dietary preferences mediate autism-gut microbiome associations. Cell 184, 5916-5931 (2021).

28. Lussu, M. et al. The urinary $1 \mathrm{H}-\mathrm{NMR}$ metabolomics profile of an Italian autistic children population and their unaffected siblings. Autism Res. 10, 1058-1066 (2017).

29. Liang, Y. et al. Untargeted metabolomic profiling using UHPLC-QTOF/MS reveals metabolic alterations associated with autism. Biomed. Res. Int. 2020:6105608 (2020) 
30. Bitar, T. et al. Identification of metabolic pathway disturbances using multimodal metabolomics in autistic disorders in a Middle Eastern population. J. Pharm. Biomed. Anal. 152, 57-65 (2018).

31. De Angelis, M. et al. Fecal microbiota and metabolome of children with autism and pervasive developmental disorder not otherwise specified. PLoS ONE 8, e76993 (2013).

32. Kang, D.-W. et al. Differences in fecal microbial metabolites and microbiota of children with autism spectrum disorders. Anaerobe 49, 121-131 (2018).

33. Wang, L. et al. Elevated fecal short chain fatty acid and ammonia concentrations in children with autism spectrum disorder. Dig. Dis. Sci. 57, 2096-2102 (2012)

34. Hsiao, E. Y. et al. Microbiota modulate behavioral and physiological abnormalities associated with neurodevelopmental disorders. Cell 155, 1451-1463 (2013).

35. Kang, D.-W. et al. Microbiota transfer therapy alters gut ecosystem and improves gastrointestinal and autism symptoms: an open-label study. Microbiome 5, 10 (2017).

36. Kang, D.-W. et al. Long-term benefit of microbiota transfer therapy on autism symptoms and gut microbiota. Sci. Rep. 9, 5821 (2019).

37. Kang, D.-W. et al. Distinct fecal and plasma metabolites in children with autism spectrum disorders and their modulation after microbiota transfer therapy. mSphere 5, e00314-20 (2020).

38. Niwa, T. et al. Oral sorbent suppresses accumulation of albumin-bound indoxyl sulphate in serum of haemodialysis patients. Nephrol. Dial. Transplant. 6, 105-109 (1991).

39. Velenosi, T. J. et al. Untargeted plasma and tissue metabolomics in rats with chronic kidney disease given AST-120. Sci. Rep. 6, 22526 (2016)

40. Schulman, G., Vanholder, R. \& Niwa, T. AST-120 for the management of progression of chronic kidney disease. Int. J. Nephrol. Renovasc. Dis. 7, 49-56 (2014).

41. Mosińska, P., Storr, M. \& Fichna, J. The role of AST-120 and protein-bound uremic toxins in irritable bowel syndrome: a therapeutic perspective. Therap. Adv. Gastroenterol. 8, 278-284 (2015).

42. Gabriele, S. et al. Urinary p-cresol is elevated in young French children with autism spectrum disorder: a replication study. Biomarkers 19, 463-470 (2014).

43. Krajmalnik-Brown, R., Lozupone, C., Kang, D.-W. \& Adams, J. B. Gut bacteria in children with autism spectrum disorders: challenges and promise of studying how a complex community influences a complex disease. Microb. Ecol. Health Dis. 26, 26914 (2015).

44. Altieri, L. et al. Urinary $p$-cresol is elevated in small children with severe autism spectrum disorder. Biomarkers 16, 252-260 (2011).

45. Gevi, F., Zolla, L., Gabriele, S. \& Persico, A. M. Urinary metabolomics of young Italian autistic children supports abnormal tryptophan and purine metabolism. Mol. Autism 7, 47 (2016).

46. Diémé, B. et al. Metabolomics study of urine in autism spectrum disorders using a multiplatform analytical methodology. J. Proteome Res. 14 5273-5282 (2015).

47. Kałużna-Czaplińska, J. Noninvasive urinary organic acids test to assess biochemical and nutritional individuality in autistic children. Clin. Biochem. 44, 686-691 (2011)

48. Bermudez-Martin, P. et al. The microbial metabolite $\mathrm{p}$-Cresol induces autistic-like behaviors in mice by remodeling the gut microbiota. Microbiome 9, 157 (2021)

49. Sun, C.-Y. et al. p-Cresol sulfate caused behavior disorders and neurodegeneration in mice with unilateral nephrectomy involving oxidative stress and neuroinflammation. Int. J. Mol. Sci. 21, 6687 (2020)

50. Karbowska, M. et al. Neurobehavioral effects of uremic toxin-indoxyl sulfate in the rat model. Sci. Rep. 10, 9483 (2020).

51. Jaglin, M. et al. Indole, a signaling molecule produced by the gut microbiota, negatively impacts emotional behaviors in rats. Front. Neurosci. 12, 216 (2018)

52. Gamage, N. et al. Human sulfotransferases and their role in chemical metabolism. Toxicol. Sci. 90, 5-22 (2006).

53. Adams, J. B., Johansen, L. J., Powell, L. D., Quig, D. \& Rubin, R. A. Gastrointestinal flora and gastrointestinal status in children with autismcomparisons to typical children and correlation with autism severity. BMC Gastroenterol. 11, 22 (2011).

54. The Pediatric Anxiety Rating Scale (PARS): development and psychometric properties. J. Am. Acad. Child Adolesc. Psychiatry 41, 1061-1069 (2002).

55. Aman, M. G., Singh, N. N., Stewart, A. W. \& Field, C. J. The aberrant behavior checklist: a behavior rating scale for the assessment of treatment effects. Am. J. Ment. Defic. 89, 485-491 (1985).

56. Constantino, John N. \& Gruber, C. P. Social Responsiveness Scale, Second Edition (Western Psychological Services, 2012).

57. Lam, K. S. L. \& Aman, M. G. The repetitive behavior scale-revised: independent validation in individuals with autism spectrum disorders. J. Autism Dev. Disord. 37, 855-866 (2007).

58. Burger-Caplan, R., Saulnier, C. A. \& Sparrow, S. S. Vineland adaptive behavior scales. In: Encyclopedia of Clinical Neuropsychology (Springer, 2018).
59. O'Donnell, L. J., Virjee, J. \& Heaton, K. W. Detection of pseudodiarrhoea by simple clinical assessment of intestinal transit rate. BMJ $\mathbf{3 0 0}$, 439-440 (1990).

60. Dimenäs, E. et al. Well-being and gastrointestinal symptoms among patients referred to endoscopy owing to suspected duodenal ulcer. Scand. J. Gastroenterol. 30, 1046-1052 (1995).

61. Nikolenko, V. N. et al. Amygdala: neuroanatomical and morphophysiological features in terms of neurological and neurodegenerative diseases. Brain Sci. 10, 502 (2020).

62. Rolls, E. T. The cingulate cortex and limbic systems for emotion, action, and memory. Brain Struct. Funct. 224, 3001-3018 (2019).

63. Guo, B. et al. Anterior cingulate cortex dysfunction underlies social deficits in Shank3 mutant mice. Nat. Neurosci. 22, 1223-1234 (2019).

64. Simms, M. L., Kemper, T. L., Timbie, C. M., Bauman, M. L. \& Blatt, G. J. The anterior cingulate cortex in autism: heterogeneity of qualitative and quantitative cytoarchitectonic features suggests possible subgroups. Acta Neuropathol. 118, 673-684 (2009).

65. Zhou, Y., Shi, L., Cui, X., Wang, S. \& Luo, X. Functional connectivity of the caudal anterior cingulate cortex is decreased in autism. PLOS ONE 11, e0151879 (2016).

66. Thakkar, K. N. et al. Response monitoring, repetitive behaviour and anterior cingulate abnormalities in autism spectrum disorders (ASD). Brain 131, 2464-2478 (2008)

67. Fishman, I., Linke, A. C., Hau, J., Carper, R. A. \& Müller, R.-A. Atypical functional connectivity of amygdala related to reduced symptom severity in children with autism. J. Am. Acad. Child Adolesc. Psychiatry 57, 764-774.e3 (2018).

68. Odriozola, P. et al. Atypical frontoamygdala functional connectivity in youth with autism. Dev. Cogn. Neurosci. 37, 100603 (2019).

69. Szekely, A., Silton, R. L., Heller, W., Miller, G. A. \& Mohanty, A. Differential functional connectivity of rostral anterior cingulate cortex during emotional interference. Soc. Cogn. Affect. Neurosci. 12, 476-486 (2016).

70. Chatham, C. H. et al. Adaptive behavior in autism: minimal clinically important differences on the Vineland-II. Autism Res. 11, 270-283 (2018).

71. Farmer, C., Adedipe, D., Bal, V. H., Chlebowski, C. \& Thurm, A.

Concordance of the Vineland Adaptive Behavior Scales, second and third editions. J. Intellect. Disabil. Res. 64, 18-26 (2020).

72. Singh, K. et al. Sulforaphane treatment of autism spectrum disorder (ASD). Proc. Natl Acad. Sci. USA 111, 15550-15555 (2014).

73. Fung, L. K. et al. Pharmacologic treatment of severe irritability and problem behaviors in autism: a systematic review and meta-analysis. Pediatrics 137, S124-S135 (2016).

74. McGuinness, G. \& Kim, Y. Sulforaphane treatment for autism spectrum disorder: a systematic review. EXCLI J. 19, 892-903 (2020).

75. Jones, R. M., Carberry, C., Hamo, A. \& Lord, C. Placebo-like response in absence of treatment in children with autism. Autism Res 10, 1567-1572 (2017).

76. Johnco, C. J. et al. Defining treatment response and symptom remission for anxiety disorders in pediatric autism spectrum disorders using the Pediatric Anxiety Rating Scale. J. Autism Dev. Disord. 45, 3232-3242 (2015).

77. Kaat, A. J., Lecavalier, L. \& Aman, M. G. Validity of the aberrant behavior checklist in children with autism spectrum disorder. J. Autism Dev. Disord. 44, 1103-1116 (2014).

78. Howes, O. D. et al. Autism spectrum disorder: consensus guidelines on assessment, treatment and research from the British Association for Psychopharmacology. J. Psychopharmacol. 32, 3-29 (2018).

79. Xiong, W. Pediatric pharmacologic management of autism-associated behavioral dysregulation. American Journal of Psychiatry Residents' Journal 12, 3-5 (2017)

80. Lord, C. et al. Autism spectrum disorder. Nat. Rev. Dis. Primers 6, 5 (2020).

81. Elbe, D. \& Lalani, Z. Review of the pharmacotherapy of irritability of autism. J. Can. Acad. Child Adolesc. Psychiatry 21, 130-146 (2012).

82. Troost, P. W. et al. Long-term effects of risperidone in children with autism spectrum disorders: a placebo discontinuation study. J. Am. Acad. Child Adolesc. Psychiatry 44, 1137-1144 (2005).

83. Nagaraj, R., Singhi, P. \& Malhi, P. Risperidone in children with autism: randomized, placebo-controlled, double-blind study. J. Child Neurol. 21, 450-455 (2006)

84. Maneeton, N. et al. Aripiprazole in acute treatment of children and adolescents with autism spectrum disorder: a systematic review and meta-analysis. Neuropsychiatr. Dis. Treat. 14, 3063-3072 (2018).

85. Marcus, R. N. et al. A placebo-controlled, fixed-dose study of aripiprazole in children and adolescents with irritability associated with autistic disorder. J. Am. Acad. Child Adolesc. Psychiatry 48, 1110-1119 (2009).

Publisher's note Springer Nature remains neutral with regard to jurisdictional claims in published maps and institutional affiliations.

(c) The Author(s), under exclusive licence to Springer Nature America, Inc. 2022 


\section{Methods}

Preclinical methods. Mouse husbandry. All animal husbandry and experiments were approved by the California Institute of Technology Institutional Animal Care and Use Committee. Throughout the study, colonized animals were maintained in autoclaved microisolator cages with autoclaved bedding (Aspen Chip Bedding, Northeastern Products), water and chow. Standard chow was provided to the animals (Laboratory Autoclavable Rodent Diet 5010, LabDiet) until diet switch to irradiated 5\% AB-2004 or control diets (Teklad) at 5 weeks. This percentage of AB-2004 (AST-120) in mouse chow has been previously used safely in mice ${ }^{34,86,87}$. Mice were maintained at an ambient temperature of $71-75^{\circ} \mathrm{F}, 30-70 \%$ humidity, at a cycle of $13 \mathrm{~h}$ light and $11 \mathrm{~h}$ dark.

Experimental design of mouse experiments. Germ-free C57BL/6J male mouse (Mus musculus) weanlings (3 weeks of age) from the Mazmanian laboratory colony were colonized by gavage of $100 \mu \mathrm{l}$ of a $1: 1$ mixture of $10^{9}$ colony-forming units per milliliter of Bacteroides ovatus ( $\pm 4 \mathrm{EP}$ pathway genes) and wild-type Lactiplantibacillus plantarum. Urine was collected at 7 weeks before behavior testing. Behavior testing began at 7 weeks of age, $3 \mathrm{~d}$ after urine collection.

Analysis of metabolites from urine of mice. Urine was passively collected, and 4EPS levels were quantified by liquid chromatography-mass spectrometry (LC-MS) and normalized to creatinine levels by Charles River Laboratories.

Behavior testing. Behavior testing was performed as previously described ${ }^{34,86,87}$. All mice were tested using the same battery of behavioral tests, starting at 6 weeks of age, in the following order: EPM, open-field testing, marble burying and grooming. Mice were allowed to settle for at least $2 \mathrm{~d}$ after cage changing before they were tested, and tests were performed $2-3 \mathrm{~d}$ apart to allow mice to rest between tests. Mice were acclimated to the behavior testing room for $1 \mathrm{~h}$ before testing. Mice were tested during the light phase of the light/dark cycle.

Clinical methods. Clinical study design and ethical approval. The AXL-2004001 study (trial registration no. ACTRN12618001956291) (ANZCTR $\left(^{88,89}\right)$ ) was an open-label, outpatient, multiple-ascending-dose phase $1 \mathrm{~b} / 2 \mathrm{a}$ study in an ASD-diagnosed adolescent (12-17 years old) population with confirmed GI symptoms (for example, diarrhea, constipation, abdominal pain and bloating). Forty-one individuals were screened between 18 April 2019 and 23 January 2020. Thirty participants were enrolled across three sites in Australia and New Zealand, including the Queensland Children's Hospital in Brisbane (14 participants), the Brain and Mind Centre in Sydney (six participants) and Optimal Clinical Trials in Auckland (ten participants). There was no formal sample size calculation for this phase 1 study because it focused on safety and tolerability. This approach is common in early-stage exploratory clinical trials. All necessary licenses and permissions to use the behavioral assessments outlined in the study protocol were obtained before initiating the study.

The study protocol, investigator brochure, participant information and consent forms, participant-facing questionnaires, recruitment documentation and procedures and documentation regarding the investigators' experience and qualifications were submitted to Health and Disability Ethics Committees (New Zealand), Children's Health Queensland Hospital and the Health Service Human Research Ethics Committee and Bellberry Human Research Ethics Committee for ethical review and approval. The study was conducted in accordance with the Declaration of Helsinki (Fortaleza, October 2013), ICH E6 guidelines, Good Clinical Practice and local regulations.

Study participation. This open-label study consisted of four different dosing plans based on participant weight at visit 1 . Eligible participants were escalated through three dosing periods during the 8-week treatment period, starting with the lowest dose for their dosing plan (see Supplementary Methods for more details). Participants were requested to consume AB-2004 90 min after any other concomitant medications. Safety and tolerability were confirmed before a participant escalated to the next dosing level. If participants were unable to tolerate a dosing level, they were returned to the previous dosing level for the remainder of the treatment period. After the last dose of AB-2004, participants returned to the clinic $28 \mathrm{~d}$ later for a follow-up safety evaluation (FV). The last visit of the study was completed on 15 May 2020. Patient data were collected using IMednet (version 1.94.0). An e-diary by Dedo (www.dedo.ai) was designed to collect GI data.

Study participants and study populations. A total of 41 adolescent individuals, aged 12-17 years inclusive, were screened for eligibility for participation in the study, and the 30 who met the study-specific eligibility criteria were enrolled and received at least one dose of AB-2004 (Safety Population). Of the 41 individuals screened and 30 enrolled, 40 and 29 , respectively, were male. A predominantly male cohort was targeted to reduce variability in response in this exploratory study that surveyed a wide range of behavioral assessments. One participant withdrew after the first dose due to the investigator's decision based on the participant presenting with an unrelated viral infection. Another participant withdrew consent during the low-dose period due to anticipated admission to hospital for pre-existing behavioral difficulties. One participant withdrew due to significant study non-compliance, and two did not complete FV assessments due to the caregivers being unwell and unable to accompany the participants. A total of 27 participants (26 males and one female) completed at least up to the EOT visit (Completers Population). One participant, the female participant, was included in the Safety Population but was not included in the exploratory efficacy analysis. This participant was removed from the exploratory efficacy analysis because their participation in the trial coincided with the initial COVID-19 pandemic outbreak and its associated societal restrictions put into effect in Australia. These restrictions prevented the participant from conducting normal routines and accessing normal services. As determined by the site principal investigator, these abrupt changes in routine had an effect on the behavior of the participant; therefore, this participant was excluded from the efficacy analysis.

Safety assessments. The primary endpoint of the study was the safety and tolerability of AB-2004 as assessed by physical examinations, vital signs, clinical laboratory measurements (hematology, serum chemistry and urinalysis) and adverse events.

Blood collection. Blood was obtained using uniform collection kits from Sonic Clinical Trials sent to each facility. Blood was drawn from study participants on visits 1, 4 and 5 and aliquoted for health monitoring by Sonic Clinical Trials and metabolite analysis by Metabolon. Blood chemistry panels performed by Sonic Clinical Trials included albumin, alkaline phosphatase, alanine amino transferase, aspartate amino transferase, blood urea nitrogen, urea, corrected calcium, bicarbonate, chloride, creatinine, gamma-glutamyl transpeptidase, glucose, lactate dehydrogenase, magnesium, phosphorus, potassium, sodium, total bilirubin, conjugated bilirubin, unconjugated bilirubin and total protein. Hematology panels included measurement of platelets, hematocrit, red blood cells, hemoglobin, reticulocytes, total white blood cell count and absolute and percentages of neutrophils, lymphocytes, monocytes, eosinophils and basophils.

Urine collection. Participants were provided with a urine home collection kit and instructions to collect all of the first morning void a maximum of $2 \mathrm{~d}$ before clinic visit and place in a refrigerator to bring to their visit or to be picked up by courier. Urinalysis samples were collected during the in-clinic visit. Aliquoting for metabolite analysis and health monitoring urinalysis was performed by Sonic Clinical Trials and included measurements of $\mathrm{pH}$, specific gravity, ketones, protein, glucose, nitrite, urobilinogen, leukocyte esterase and blood.

Human plasma metabolite quantification. Human plasma was analyzed by Metabolon. In brief, plasma was spiked with internal standards (4-ethylphenyl sulfate- $\mathrm{d}_{4}, \mathrm{p}$-cresol sulfate- $\mathrm{d}_{7}, 3$-hydroxyhippurate- ${ }^{13} \mathrm{C}_{2},{ }^{15} \mathrm{~N}$, 3-hydroxyphenylacetate- $\mathrm{d}_{3}, 3$-(3-hydroxyphenyl)-3-hydroxypropionate- $\mathrm{d}_{3}$, 3-indoxyl sulfate- ${ }^{13} \mathrm{C}_{6}, 3$-(4-hydroxyphenyl)propionate- $\mathrm{d}_{4}, \mathrm{p}$-cresol glucuronide- $\mathrm{d}_{7}$ and $\mathrm{N}$-acetylserine- $\mathrm{d}_{3}$,), protein precipitated and analyzed on an Agilent 1290/ AB Sciex 5500 QTrap LC-MS/MS system equipped with a UHPLC C18 column. Quantitation was performed using a weighted linear least squares regression analysis with a weighting of $1 / x$ or $1 / x^{2}$ generated from fortified calibration standards prepared immediately before each run.

Human urine metabolite quantification. Human urine was analyzed by Metabolon. In brief, urine was diluted ten-fold and spiked with internal standards (p-cresol sulfate- $\mathrm{d}_{7}, 3$-hydroxyhippurate- ${ }^{13} \mathrm{C}_{2},{ }^{15} \mathrm{~N}, 3$-hydroxyphenylacetate- $\mathrm{d}_{3}$, 3-(3-hydroxyphenyl)-3-hydroxypropionate- $\mathrm{d}_{3}, 3$-indoxyl sulfate- ${ }^{-13} \mathrm{C}_{6}, 3$ (4-hydroxyphenyl)propionate- $\mathrm{d}_{4}$, $\mathrm{p}$-cresol glucuronide- $\mathrm{d}_{7}$ and $\mathrm{N}$-acetylserine- $\mathrm{d}_{3}$ ), and then an aliquot was subjected to either a solvent crash (for $\mathrm{p}$-cresol sulfate, 3-indoxyl sulfate and p-cresol glucuronide) or derivatization (for 3-hydroxyhippurate, 3-hydroxyphenylacetate, 3-(3-hydroxyphenyl)-

3-hydroxypropionate, $\mathrm{N}$-acetylserine and 3-(4-hydroxyphenyl)propionate) and analyzed on an Agilent 1290/AB Sciex 5500 QTrap LC-MS/MS system equipped with a UHPLC C18 column in negative mode. Quantification of 4EPS was performed by the same method with a solvent crash (using the internal standard, 4-ethylphenyl sulfate- $\mathrm{d}_{4}$ ) but without sample dilution. Quantitation was performed using a weighted linear least squares regression analysis with a weighting of $1 / x$ generated from fortified calibration standards prepared immediately before each run. All urine metabolites were normalized to creatinine levels.

Exploratory efficacy assessments. Exploratory efficacy outcomes included changes from BL at EOT and FV on the GSI-6, Numerical Rating Scale (NRS), GSRS, BSS, RBS-R VABS, CASI-5, SRS, CGI-S and CGI-I, ABC or PARS diagnostics. Efficacy assessments were administered on site at the respective clinics during visits. VABS, PARS and CGI-S and CGI-I were conducted by the principal investigator or qualified designee. The GSI-6, NRS, GSRS, RBS-R, BSS, CASI-5 SRS and ABC questionnaires were completed by the designated caregivers of the participants. In the VABS assessment, ten participants did not pass the under $25 \%$ estimated answers criterion of any domain during assessment and, thus, had to be removed from this analysis, according to the VABS manual, page 47 (ref. ${ }^{5}$ ). 
$A B$-2004 treatment dosage. For individuals weighing $\geq 60 \mathrm{~kg}$, three daily doses each of:

- Period 1: 0.75g, Days 1-14 (2 weeks)

- Period 2: 1.5 g, Days 15-28 (2 weeks)

- $\quad$ Period 3: 2g, Days 29-56 (4 weeks)

For individuals weighing 50-59 kg, three daily doses each of:

- $\quad$ Period 1: 0.75g, Days 1-14 (2 weeks)

- Period 2: 1.0 g, Days 15-28 (2 weeks)

- Period 3: 1.75g, Days 29-56 (4 weeks)

For individuals weighing $40-49 \mathrm{~kg}$, three daily doses each of:

- Period 1: 0.5 g, Days 1-14 (2 weeks)

- Period 2: 0.75g, Days 15-28 (2 weeks)

- $\quad$ Period 3: 1.5g, Days 29-56 (4 weeks)

For individuals weighing $30-39 \mathrm{~kg}$, three daily doses each of:

- $\quad$ Period 1: 0.5 g, Days 1-14 (2 weeks)

- Period 2: 0.75g, Days 15-28 (2 weeks)

- $\quad$ Period 3: 1.0g, Days 29-56 (4 weeks)

Magnetic resonance. Scan parameters. All scans were collected using phased array receive-only head coils (32 channels at sites 1 and 2, 64 channels at site 3).

High-resolution anatomic images (T1-weighted (T1w) and T2-weighted $(\mathrm{T} 2 \mathrm{w}))$ were acquired with 1-mm isotropic resolution. T1w images (2@4:01 each, for sites 1 and 2, and 1@4:00 for site 3) were sagittally oriented using a 3D MPRAGE sequence. A single resolution matched T2w image (4:28) was acquired (the T2_CUBE sequence at site 1, T2_SPACE at sites 2 and 3).

Two gradient echo multi-band echo planar imaging (EPI) rs-FMRI acquisitions (300 volumes each) were performed with 2.5 - $\mathrm{mm}$ isotropic resolution, 1 - $\mathrm{s}$ repetition time and multi-band factor 3 . In total, 51 slices were acquired obliquely, with the bottom slice oriented on the line between the bottom of the cerebellum and the bottom of the orbitofrontal cortex. The phase encode was reversed between the first and second scan (AP for the first scan, PA for the second) to allow for distortion correction.

Two diffusion scans were also acquired as part of the protocol (5:56 each), but they were not used for this analysis.

Data processing. Before processing, all data were named and organized following the BIDS 1.2.1 specification. Anatomical and fMRI data used in this manuscript were pre-processed using fMRIPrep 20.0.4 (ref. ${ }^{90}$ ) (RRID: SCR_016216), which is based on Nipype 1.4.2 (ref. ${ }^{91}$ ) (RRID: SCR_002502).

Anatomical data pre-processing. A total of two T1w images were corrected for intensity non-uniformity (INU) with N4BiasFieldCorrection ${ }^{92}$, distributed with ANTs 2.2.0 (ref. ${ }^{93}$ ) (RRID: SCR_004757) per individual. The T1w reference was then skull-stripped with a Nipype implementation of the antsBrainExtraction. shworkflow (from ANTs), using OASIS30ANTs as target template. Brain tissue segmentation of cerebrospinal fluid (CSF), white matter (WM) and gray matter (GM) was performed on the brain-extracted T1w using fast (FSL 5.0.9, RRID: SCR_002823) $)^{94}$. A T1w reference map was computed after registration of four T1w images (after INU correction) using mri_robust_template (FreeSurfer 6.0.1) ${ }^{95}$. Brain surfaces were reconstructed using recon-all (FreeSurfer 6.0.1, RRID: SCR_001847 $)^{96}$, and the brain mask estimated previously was refined with a custom variation of the method to reconcile ANTs-derived and FreeSurfer-derived segmentations of the cortical GM of Mindboggle (RRID: SCR_002438 $)^{97}$. Volume-based spatial normalization to two standard spaces (MNI152NLin6Asym and MNI152NLin2009cAsym) was performed through non-linear registration with antsRegistration (ANTs 2.2.0), using brain-extracted versions of both $\mathrm{T} 1 \mathrm{w}$ reference and the $\mathrm{T} 1 \mathrm{w}$ template. The following templates were selected for spatial normalization: FSL's MNI ICBM 152 non-linear 6th Generation Asymmetric Average Brain Stereotaxic Registration Model $^{98}$ (RRID: SCR_002823; TemplateFlow ID: MNI152NLin6Asym) and ICBM 152 Nonlinear Asymmetrical template version 2009c ${ }^{98,99}$ (RRID:SCR_008796; TemplateFlow ID: MNI152NLin2009cAsym)

Functional data pre-processing. For each of the four blood oxygenation level dependent (BOLD) runs found per participant (across all tasks and sessions), a reference volume and its skull-stripped version were generated using fMRIPrep. A deformation field to correct for susceptibility distortions was estimated based on fMRIPrep's fieldmap-less approach. Registration is performed with antsRegistration (ANTs 2.2.0), and the process is regularized by constraining deformation to be non-zero only along the phase-encoding direction and modulated with an average fieldmap template ${ }^{100}$. A corrected EPI reference was calculated. The BOLD reference was then co-registered to the T1w reference using bbregister (FreeSurfer) ${ }^{101}$. Co-registration was configured with 6 degrees of freedom. Head motion parameters with respect to the BOLD reference were estimated before any spatiotemporal filtering using mcflirt (FSL 5.0.9) ${ }^{102}$. BOLD runs were slice time corrected using 3dTshift from AFNI 20160207 (ref. ${ }^{103}$ ) (RRID: SCR_005927). The BOLD time series were resampled onto their original, native space by applying a single composite transform to correct for head motion and susceptibility distortions. The BOLD time series were resampled into standard space, generating a pre-processed BOLD run in MNI152NLin6Asym space. First, a reference volume and its skull-stripped version were generated using a custom methodology of fMRIPrep. Automatic removal of motion artifacts using independent component analysis (ICA-AROMA) ${ }^{104}$ was performed on the pre-processed BOLD on MNI space time series after removal of non-steady-state volumes and spatial smoothing with an isotropic, Gaussian kernel of $6 \mathrm{~mm}$ full width at half maximum. Corresponding 'non-aggressively' de-noised runs were produced after such smoothing. Additionally, the 'aggressive' noise regressors were collected and placed in the corresponding confounds file. Several confounding time series were calculated based on the pre-processed BOLD: framewise displacement (FD), DVARS and three region-wise global signals. FD and DVARS were calculated for each functional run, both using their implementations in Nipype (following the definitions by Power et al. ${ }^{105}$ ). The three global signals were extracted within the CSF, the WM and the whole brain masks. Additionally, a set of physiological regressors was extracted to allow for component-based noise correction (CompCor) ${ }^{106}$. Principal components were estimated after high-pass filtering the pre-processed BOLD time series (using a discrete cosine filter with 128-s cutoff) for the two CompCor variants: temporal (tCompCor) and anatomical (aCompCor). tCompCor components were then calculated from the top 5\% variable voxels within a mask covering the subcortical regions. This subcortical mask was obtained by heavily eroding the brain mask. For aCompCor, components were calculated within the intersection of the mask and the union of CSF and WM masks calculated in T1w space, after their projection to the native space of each functional run (using the inverse BOLD-to-T1w transformation). Components were also calculated separately within the WM and CSF masks. For each CompCor decomposition, the $k$ components with the largest singular values were retained, such that the retained components' time series were sufficient to explain $50 \%$ of variance across the nuisance mask (CSF, WM, combined or temporal). The head motion estimates calculated in the correction step were also placed within the corresponding confounds file. The confound time series derived from head motion estimates and global signals were expanded with the inclusion of temporal derivatives and quadratic terms for each ${ }^{107}$. Frames that exceeded a threshold of $0.5 \mathrm{~mm}$ FD or 1.5 standardized DVARS were annotated as motion outliers. Gridded (volumetric) resamplings were performed using antsApplyTransforms (ANTs) and configured with Lanczos interpolation to minimize the smoothing effects of other kernels ${ }^{107}$. Non-gridded (surface) resamplings were performed using mri_vol2surf (FreeSurfer).

fMRI data analysis. To quantify connectivity between the bilateral amygdala and rACC, a region of interest (ROI) approach was used employing methods from our previous work $^{108}$. The bilateral amygdala was defined using the Harvard-Oxford atlas. The rACC ROI is just anterior to the genu of the corpus callosum and was used in our previous work ${ }^{108,109}$. Average time courses for each ROI were extracted, demeaned, detrended, Hamming windowed and correlated to generate a single correlation value $(r)$ for each participant both before and after treatment.

Statistical information. Results presented here are from post hoc analyses of the data from the clinical trial using GraphPad Prism 9. Here we present bar graphs representing the preclinical data by mean \pm s.e.m. analyzed by ordinary two-way ANOVA test with false discovery rate correction using the Benjamini, Krieger and Yekutieli method, with individual variances computed for each comparison. Clinical data are presented as mean, and 95\% confidence intervals were analyzed by repeated-measures ANOVA or linear mixed-effects model, with Geisser-Greenhouse correction tests and false discovery rate correction by the Benjamini, Krieger and Yekutieli method. Metabolite data are presented as individual graphs but were statistically analyzed across all metabolites and samples. Clinical behavioral metrics were analyzed within each test. Two-tailed Pearson's correlations were performed comparing change in metabolite levels to change in behavioral scores for the PARS and ABC-I tests. fMRI values were analyzed using a two-tailed paired $t$-test. Study participants were studied as a single group, and all comparisons, especially those within the subgroup of participants in the top quartile of ASD severity, were post hoc and exploratory in nature. Missing data were not imputed, and data were analyzed for individuals who withdrew from the study, for any reason before study completion, regardless of treatment duration, up to the point of discontinuation.

Reporting Summary. Further information on research design is available in the Nature Research Reporting Summary linked to this article.

\section{Data availability}

Data generated or analyzed during this study are included in this published article, and individual de-identified participant data can be found in the Supplementary Information. Axial Therapeutics ('Axial'), under certain restrictions below, will provide access to at least the minimum datasets from its clinical trials that are necessary to interpret, verify and extend the research findings. Axial considers that the minimum datasets might include the study protocol (already provided as Supplementary Data) and the tables, lists and figures from the final Clinical Study Report. Such datasets will be made available only to qualified scientific researchers for legitimate scientific research purposes. Simultaneously, Axial is 
mandated by law and otherwise is committed to protecting the privacy and rights of all individuals who participate in its clinical trials; therefore, all data supplied will be de-identified according to applicable regulations. Data requests should be sent to the corresponding author, A. Stewart Campbell, at stewart@axialtx.com (subject line: 'AB-2004 Clinical Trial Data Request'). All data requests must be clearly described in a written proposal, including statement of research purpose, research plan and methods (including statistical analysis plan, if applicable), key research personnel and data sharing plans. All requests meeting submission requirements will be promptly considered for scientific merit by internal and external (if necessary) subject matter experts and compliance personnel, and a written response approving or denying access to the data will be provided. Approved requests will result in access to the necessary data through a secure data sharing portal, and access will be granted for a pre-specified defined period of time that is commensurate with the research plan. Under approved requests, Axial and the interested party must enter a data sharing agreement that will govern the terms and conditions for use, storage and communication of the data and terms for co-authorship resulting from any publication of the research results. It is recognized that certain regulations might apply in different countries, states or regions that might affect what data may be shared and with whom; Axial cannot guarantee access to the requested data in these circumstances. Source data are provided with this paper.

\section{References}

86. Sharon, G. et al. Human gut microbiota from autism spectrum disorder promote behavioral symptoms in mice. Cell 177, 1600-1618 (2019).

87. Komada, M., Takao, K. \& Miyakawa, T. Elevated plus maze for mice. J. Vis. Exp. 1088 (2008).

88. Esteban, O. et al. fMRIPrep: a robust preprocessing pipeline for functional MRI. Nat. Methods 16, 111-116 (2019)

89. Esteban, O. et al. nipy/nipype: 1.5.1. https://zenodo.org/record/4035081 (2020).

90. Gorgolewski, K. et al. Nipype: a flexible, lightweight and extensible neuroimaging data processing framework in Python. Front. Neuroinform. 5, 13 (2011).

91. Tustison, N. J. et al. N4ITK: improved N3 bias correction. IEEE Trans. Med. Imaging 29, 1310-1320 (2010).

92. Avants, B. B., Epstein, C. L., Grossman, M. \& Gee, J. C. Symmetric diffeomorphic image registration with cross-correlation: evaluating automated labeling of elderly and neurodegenerative brain. Med. Image Anal. 12, 26-41 (2008)

93. Zhang, Y., Brady, M. \& Smith, S. Segmentation of brain MR images through a hidden Markov random field model and the expectation-maximization algorithm. IEEE Trans. Med. Imaging 20, 45-57 (2001).

94. Reuter, M., Rosas, H. D. \& Fischl, B. Highly accurate inverse consistent registration: a robust approach. Neuroimage 53, 1181-1196 (2010).

95. Dale, A. M., Fischl, B. \& Sereno, M. I. Cortical surface-based analysis: I. Segmentation and surface reconstruction. Neuroimage $\mathbf{9}$, 179-194 (1999).

96. Klein, A. et al. Mindboggling morphometry of human brains. PLoS Comput. Biol. 13, e1005350 (2017).

97. Evans, A. C., Janke, A. L., Collins, D. L. \& Baillet, S. Brain templates and atlases. Neuroimage 62, 911-922 (2012).

98. Fonov, V., Evans, A., McKinstry, R., Almli, C. \& Collins, D. Unbiased nonlinear average age-appropriate brain templates from birth to adulthood. Neuroimage 47, S102 (2009).

99. Treiber, J. M. et al. Characterization and correction of geometric distortions in 814 diffusion weighted images. PLoS ONE 11, e0152472 (2016).

100. Greve, D. N. \& Fischl, B. Accurate and robust brain image alignment using boundary-based registration. Neuroimage 48, 63-72 (2009).
101. Jenkinson, M., Bannister, P., Brady, M. \& Smith, S. Improved optimization for the robust and accurate linear registration and motion correction of brain images. Neuroimage 17, 825-841 (2002).

102. Cox, R. W. \& Hyde, J. S. Software tools for analysis and visualization of fMRI data. NMR Biomed. 10, 171-178 (1997).

103. Pruim, R. H. R. et al. ICA-AROMA: a robust ICA-based strategy for removing motion artifacts from fMRI data. Neuroimage 112, 267-277 (2015).

104. Power, J. D. et al. Methods to detect, characterize, and remove motion artifact in resting state fMRI. Neuroimage 84, 320-341 (2014).

105. Behzadi, Y., Restom, K., Liau, J. \& Liu, T. T. A component based noise correction method (CompCor) for BOLD and perfusion based fMRI. Neuroimage 37, 90-101 (2007).

106. Satterthwaite, T. D. et al. An improved framework for confound regression and filtering for control of motion artifact in the preprocessing of resting-state functional connectivity data. Neuroimage 64, 240-256 (2013).

107. Lanczos, C. Evaluation of noisy data. J. Soc. Ind. Appl. Math. B Numer. Anal. 1, 76-85 (1964).

108. Janes, A. C. et al. Nicotine normalizes cortico-striatal connectivity in non-smoking individuals with major depressive disorder. Neuropsychopharmacology 43, 2445-2451 (2018).

109. McCarthy, J. M. et al. Reduced interhemispheric executive control network coupling in men during early cocaine abstinence: a pilot study. Drug Alcohol Depend. 181, 1-4 (2017).

\section{Acknowledgements}

We acknowledge the study participants and their families for their generous contributions of time and effort to this study. This work was supported by funds from Axial Therapeutics and was conducted by, and in collaboration with, the company.

\section{Author contributions}

Conceptualization: A.S.C., B.D.N., D.H.D. and S.K.M. Preclinical methodology: B.D.N and S.K.M. Clinical methodology: A.S.C., C.R.M., M.C., G.M.P. and S.R. Formal analysis: B.D.N., C.R.M., G.M.P., A.C.J. and B.F. Preclinical investigation: B.D.N. Clinical investigation: A.S.C., C.R.M., J.T., G.M.P., F.B., H.H., R.G., A.G., A.C.J. and B.F. Resources: Axial Therapeutics. Writing-original draft: B.D.N. Writing-review and editing: A.S.C., B.D.N., C.R.M., F.B. and S.K.M. Visualization: B.D.N. Preclinical supervision: S.K.M. Clinical supervision: A.S.C. Project administration: B.D.N. and C.R.M. Funding acquisition: Axial Therapeutics.

\section{Competing interests}

A.S.C., C.R.M., M.C., G.M.P., F.B., S.R., D.H.D. and S.K.M. have financial interests in Axial Therapeutics. J.T., A.C.J. and B.F. were paid consultants of Axial Therapeutics. H.H., R.G. and A.J.G. are investigators at clinics contracted by Axial Therapeutics. B.D.N. declares no competing interests.

\section{Additional information}

Extended data is available for this paper at https://doi.org/10.1038/s41591-022-01683-9. Supplementary information The online version contains supplementary material available at https://doi.org/10.1038/s41591-022-01683-9.

Correspondence and requests for materials should be addressed to A. Stewart Campbell or Sarkis K. Mazmanian.

Peer review information Nature Medicine thanks Ruth Luna and the other, anonymous, reviewer(s) for their contribution to the peer review of this work. Jerome Staal was the primary editor on this article and managed its editorial process and peer review in collaboration with the rest of the editorial team.

Reprints and permissions information is available at www.nature.com/reprints. 
a
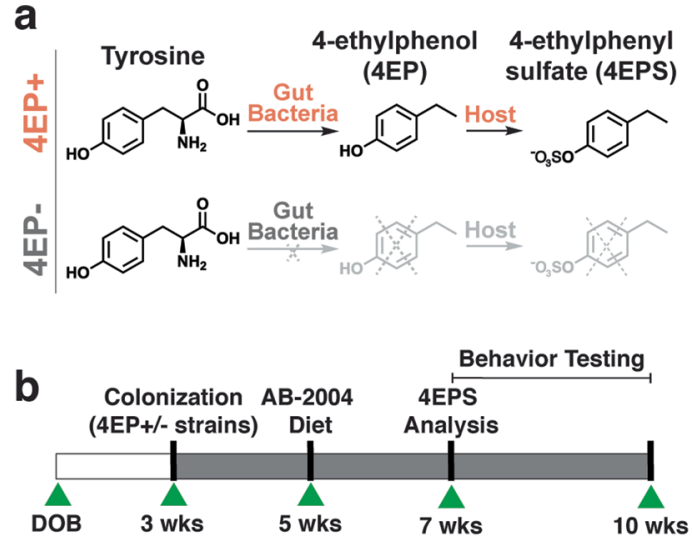

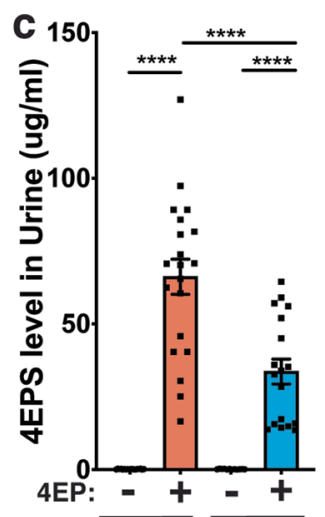

Diet: $\overline{\text { Control }} \overline{\mathrm{AB}-2004}$
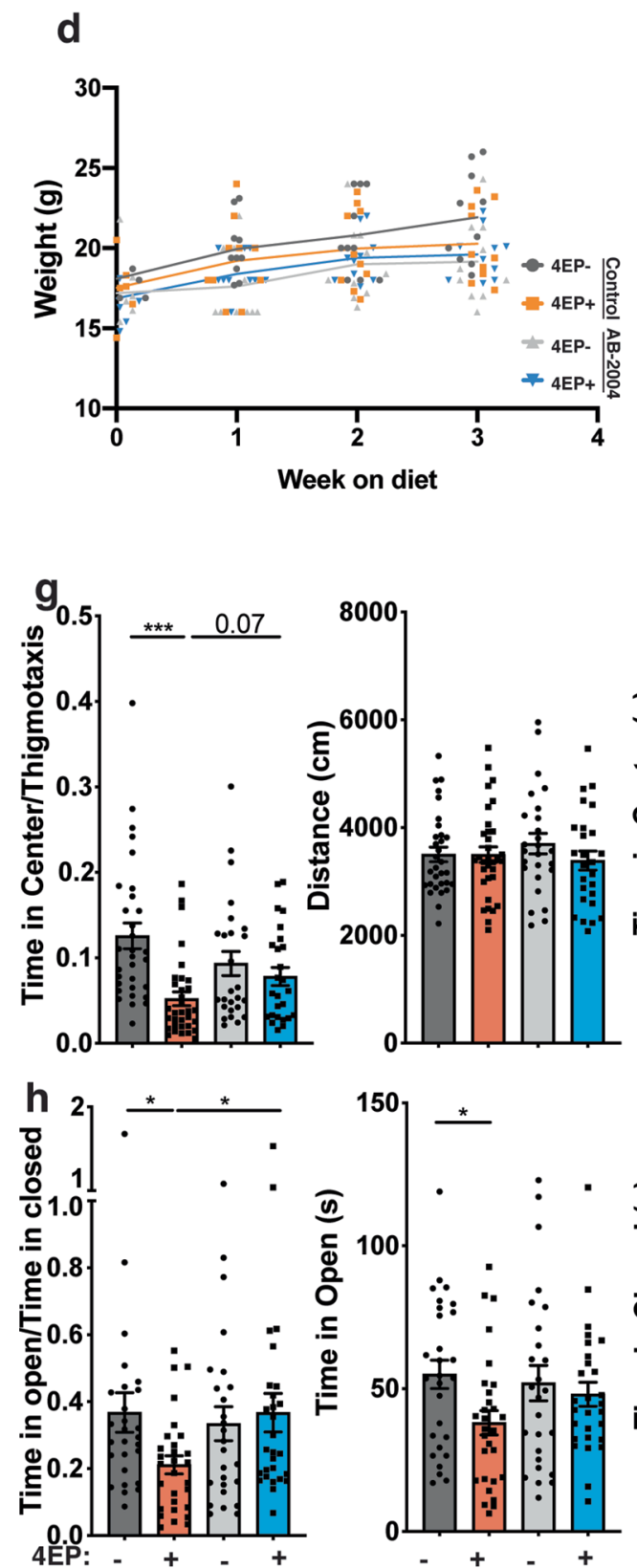

Diet: $\frac{-\quad+}{\text { Control }} \frac{-+}{\mathrm{AB}-2004}$

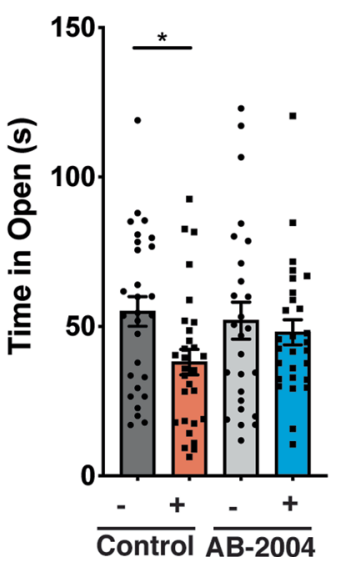

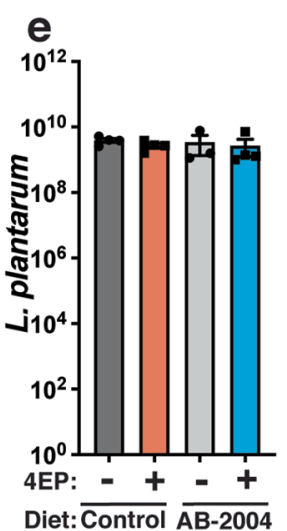

Diet: $\overline{\text { Control }} \overline{\mathrm{AB}-2004}$

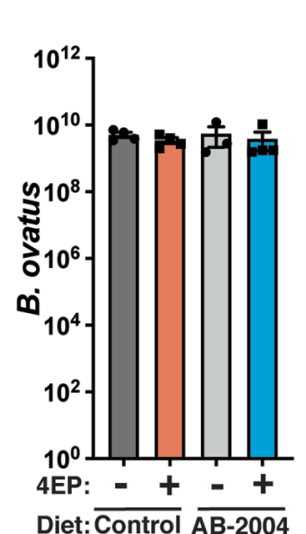

f
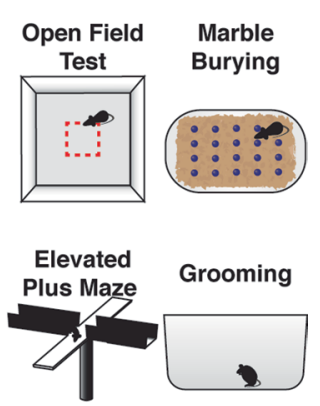
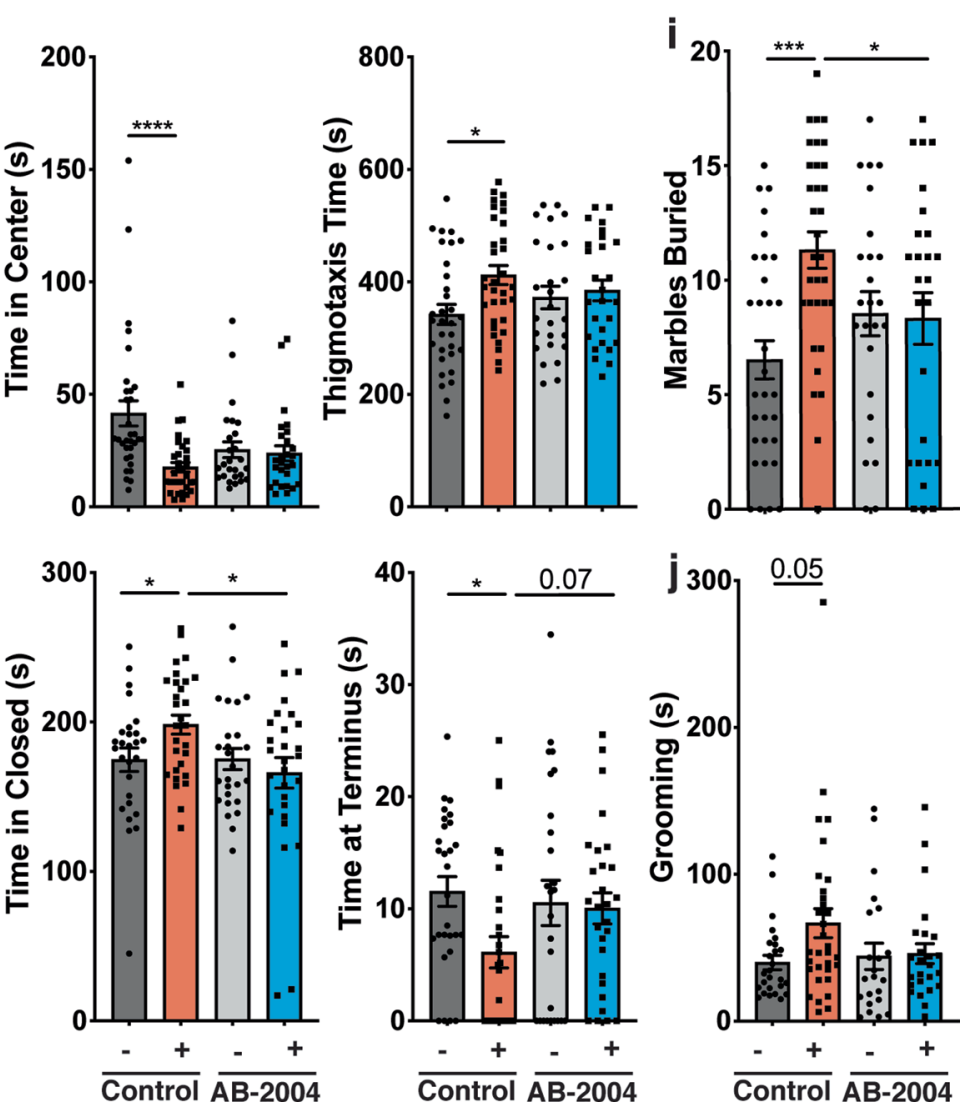

Extended Data Fig. 1 | See next page for caption. 
Extended Data Fig. 1 | AB-2004 administration lowers 4EPS levels in gnotobiotic mice and ameliorates anxiety-like behavior. a, Bacterial strain pairs were engineered to produce 4-ethylphenol (4EP+) or not (4EP-). See Methods and [see accompanying manuscript, accepted for publication] for details. $4 E P$ is converted to 4EPS by the mouse. $\mathbf{b}$, Timeline schematic for colonization of germ-free mice, AB-2004 administration, and metabolite and behavioral analysis. c, Separate groups of mice were each colonized with either the 4EP+ or 4EP- strain pair. 4EPS levels quantified in urine of mice two weeks after dietary administration of AB-2004 or control diets. Limit of detection is $10 \mathrm{ng} / \mathrm{ml}$ ( $\mathrm{n}$ number left to right: $\mathrm{n}=18,20,12,18$ ). All adj. $p$-values, $<0.0001$. d, Weight gain of mice on diet containing $5 \%$ AB-2004 compared to controls ( $n=10$ each). e, Colonization of mice with 4EP producing strain pairs of Lactobacillus plantarum and Bacteroides ovatus. Both bacterial strains equally colonize 4EP+ and 4EP- groups of mice, independent of diet ( $n$ number left to right: $n=4,4,3,4)$. $\mathbf{f - j}$, Behavioral test results from mice administered AB-2004 or control diet. $\mathbf{f}$, Visual representations of the behavioral assays open field, elevated plus maze, marble burying and grooming. $\mathbf{g}$, Open field (OF) test results presented as, from left to right, a ratio of time spent in the center of the arena over time spent in thigmotaxis along the perimeter during the 10-minute testing period, total distance mice traveled, time in center, and time in thigmotaxis along the perimeter of the arena (n number left to right: $n=31,33,26,26$ ). Exact adj. $p$-values: center/thigmotaxis, $0.0003 ;$ center, $<0.0001$; thigmotaxis, 0.03. h, Elevated plus maze (EPM) results presented, from left to right, as a ratio of time spent in the open arms of the maze over time spent in the closed arms during the 5-minute testing period, time mice spent in the open arms, time spent in the closed arms, and total time at the terminus (outermost third of open arms) ( $n$ number left to right: $n=27,29,26,28$ ). Exact adj. $p$-values: open/closed, both 0.014; open, 0.03; closed, 0.045, 0.01; terminus, 0.01. i, Number of marbles buried during the 10-minute testing period ( $n$ number left to right: $n=31,33,25,26$ ). Exact adj. $p$-values: 0.0006 ; 0.04. j, Amount of time spent grooming during the 10-minute testing period ( $n$ number left to right: $n=26,32,22,25$ ). Abbreviations: 4EP, 4-ethylphenol; 4EPS, 4-ethylphenyl sulfate. Data represent mean \pm SEM analyzed by ordinary two-way ANOVA test with FDR correction, with individual variances computed for each comparison ( ${ }^{\star} p$ adj. $\leq 0.05 ;{ }^{\star \star} p$ adj. $\leq 0.01 ;{ }^{\star \star \star} p$ adj. $\leq 0.001 ;{ }^{\star \star \star \star} p$ adj. $\leq 0.0001$ ). 


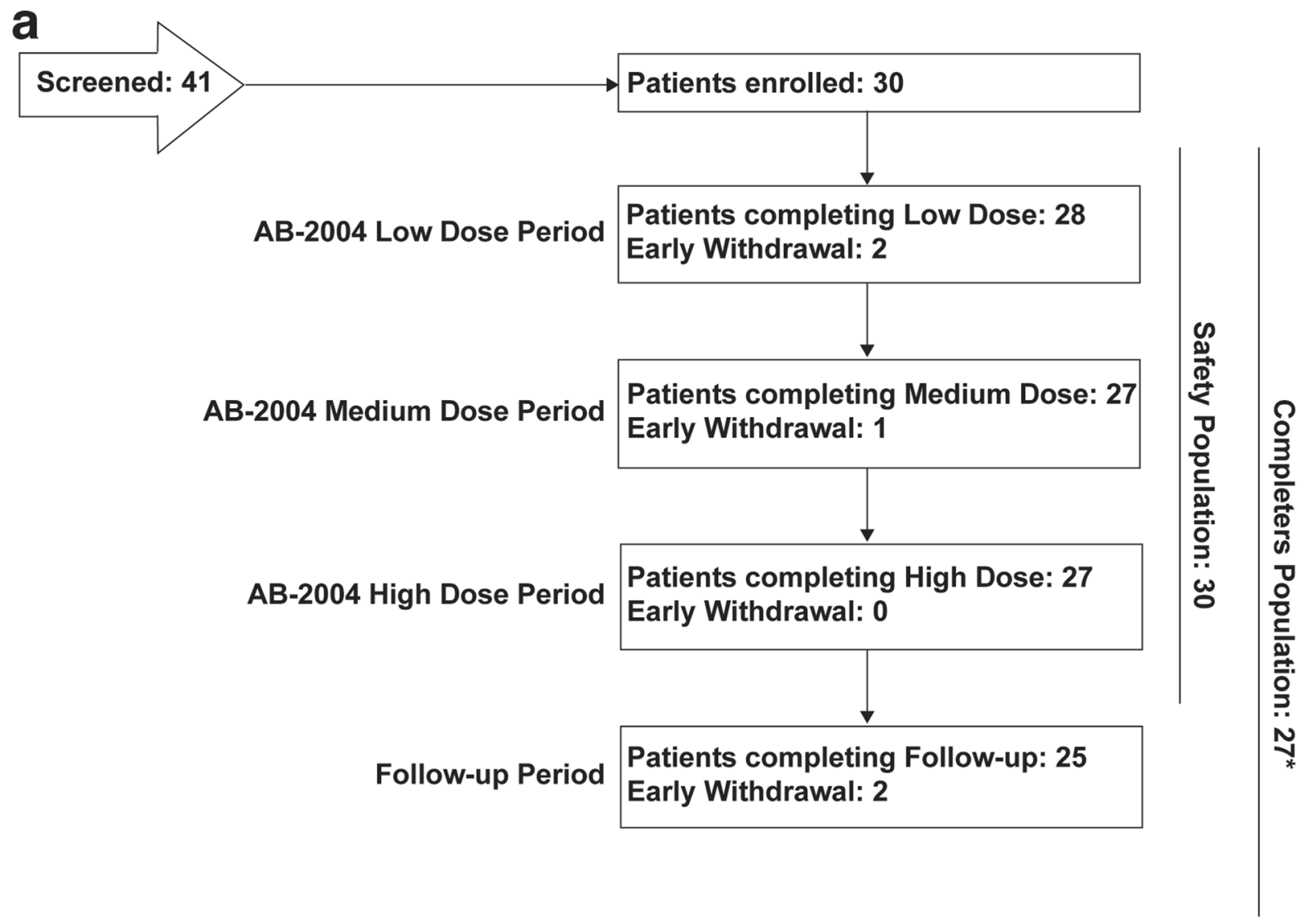

*One subject removed from analysis here due to COVID19 schedule interruptions

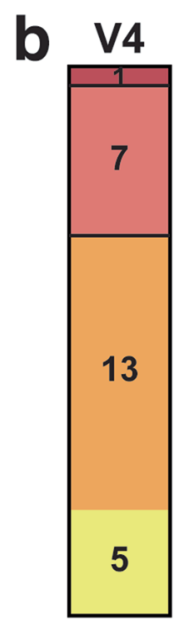

Total=26
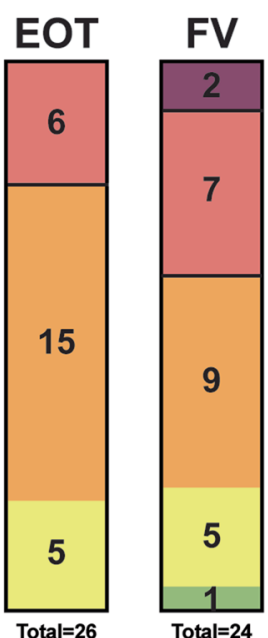
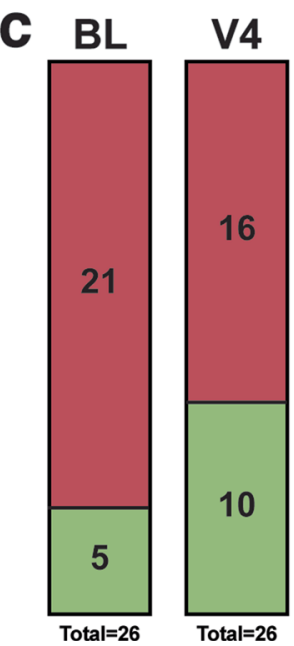

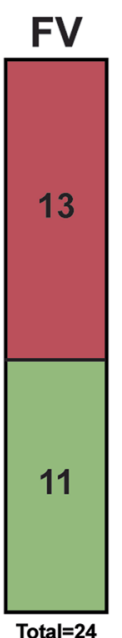

GI Disorder

No GI or

Borderline No GI Disorder

Extended Data Fig. 2 | Consort flow diagram, CGI-S, and CGI-I data in clinical samples. a, A total of 41 individuals were screened for eligibility across 3 sites in New Zealand and Australia between April 2019 and January 2020. 30 participants were enrolled following meeting predefined criteria for study (see methods and Extended Data Table 1). 27 participants completed treatment and 25 completed the follow up visit. b, Clinical global impression improvement scores (CGI-I) for all participants at visit 4, EOT and FV time points ( $n=26$ with two missing participants for FV due to parental illness). All participants' scores were normalized to a 4 at BL, thus this data is relative to 4 ; lower number $=$ improvement, higher number $=$ worsening. $\mathbf{c}$, Clinical global impression severity (CGI-S) score distribution for completers group at baseline (BL), visit 2, visit 4, end of treatment (EOT), and final visit (FV) time points. Colors are defined according to legend, with green including both absence of $\mathrm{Gl}$ symptoms and borderline $\mathrm{Gl}$ symptoms ( $\mathrm{n}=26 \mathrm{with}$ two missing participants for FV due to parental illness). Abbreviations: CONSORT, Consolidated Standards of Reporting Trials; CGI-S, clinical global impression severity; CGI-I, clinical global improvement; rACC2, rostral anterior cingulate cortex; BL, baseline; V2, visit 2; V4, visit 4; EOT, end of treatment; FV, final visit. 
a
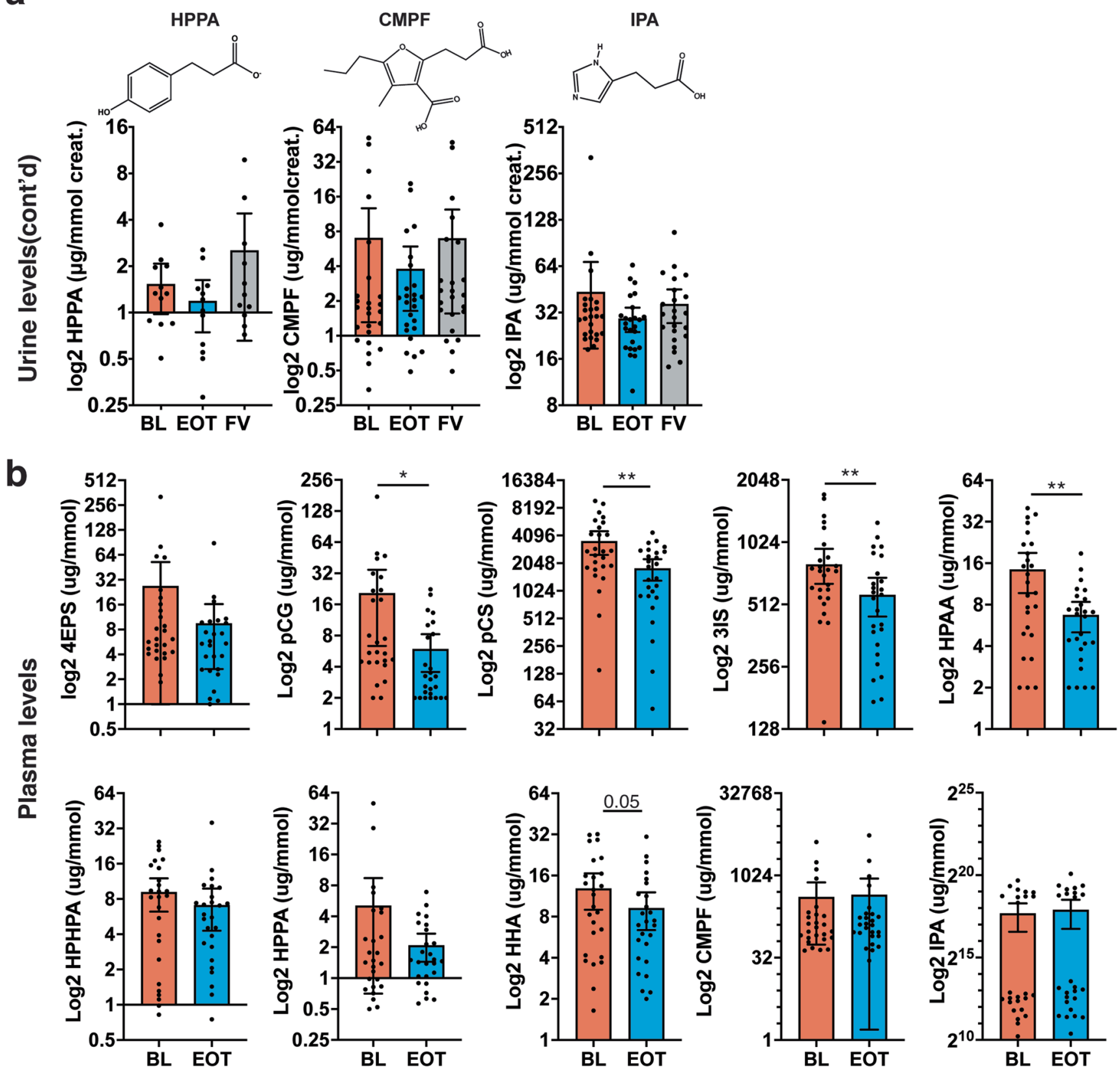

C

\begin{tabular}{|l|c|c|c|c|}
\hline & \multicolumn{2}{|c|}{ BL } & \multicolumn{2}{c|}{ EOT } \\
\hline & Pearson's r & $p$-value & Pearson's r & $p$-value \\
\hline 4EPS & 0.81 & $<0.0001$ & 0.96 & $<0.0001$ \\
\hline pCG & 0.86 & $<0.0001$ & 0.84 & $<0.0001$ \\
\hline pCS & 0.68 & 0.0001 & 0.75 & $<0.0001$ \\
\hline 3IS & 0.51 & 0.0078 & 0.23 & 0.27 \\
\hline HPAA & 0.32 & 0.11 & 0.84 & $<0.0001$ \\
\hline HPHPA & 0.12 & 0.57 & 0.53 & 0.0064 \\
\hline HPPA & -0.04 & 0.86 & 0.24 & 0.24 \\
\hline HHA & 0.32 & 0.11 & 0.59 & 0.0019 \\
\hline CMPF & 0.90 & $<0.0001$ & 0.82 & $<0.0001$ \\
\hline IPA & 0.14 & 0.52 & 0.24 & 0.25 \\
\hline
\end{tabular}
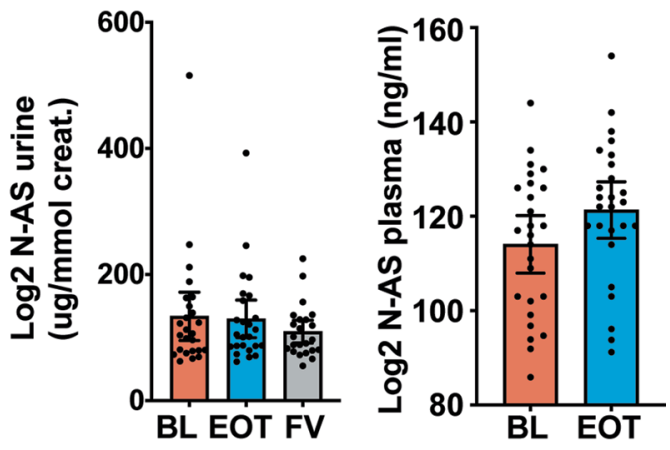

Extended Data Fig. 3 | See next page for caption. 
Extended Data Fig. 3 | Quantitative values of metabolites in plasma and correlations between urine and plasma metabolite levels. a, Metabolite levels in urine that were not altered from baseline (BL, orange), end of treatment (EOT, blue), and final visit (FV, grey) timepoints from the completers group, normalized to creatinine ( $\mu \mathrm{g}$ metabolite/mmol creatinine) on log2 scale. Chemical structures shown above associated data panels. Urine from one subject could not be obtained due to incontinence, and two participants did not complete the FV timepoint due to parental illness $(B L, E O T n=25 ; F V n=23)$. HPPA measured below the limit of quantification in $>50 \%$ of individuals; no imputation was performed, missing values not shown. $\mathbf{b}$, Quantitative values of metabolites in plasma samples at baseline (BL) and end of trial (EOT) timepoints with $n g / m l$ levels (log2 scale) along the $y$-axis $(n=26)$. Exact p-values: pCG, 0.04; pCS, 0.003; 3IS, 0.005; HPAA, 0.005. c, Pearson correlation between plasma $(\mathrm{ng} / \mathrm{ml})$ and urine (ug/mmol creatinine) levels for each measured metabolite $(n=25)$. d, Quantitative values of control metabolite, $N$-acetyl serine $(N-A S)$ in urine and plasma samples (left panel: $B L, E O T n=25$; $F V n=23$ ) (right panel: $n=26)$. Abbreviations: BL, baseline; EOT, end of treatment; HPPA, 3-(4-hydroxyphenyl)propionate; HHA, 3-hydroxyhippurate; CMPF, 3-carboxy-4-methyl-5-propyl-2-furanpropanoate; IPA, imidazolepropionate; 4EPS, 4-ethylphenyl sulfate; pCG, p-cresol glucuronide; pCS; p-cresol sulfate; 3IS, 3-indoxyl sulfate; HPHPA, 3-(3-hydroxyphenyl)-3- hydroxypropionate; HPPA, 3-(4-hydroxyphenyl)propionate; HHA, 3-hydroxyhippurate; HPAA, 3-hydroxyphenylacetate; N-AS, N-acetyl serine. Data analysis was conducted on the completers group, with each sample representing a separate study participant. Data analysis is exploratory and post hoc in nature, shown as mean and $95 \%$ confidence intervals in panels a and c, analyzed by a twotailed paired t-test or ANOVA with multiple comparisons and false discovery rate correction as appropriate $\left({ }^{\star} p \leq 0.05 ;{ }^{\star \star} p \leq 0.01 ;{ }^{* \star \star} p \leq 0.001\right)$ and a Pearson's correlation in panel $b$. 
a
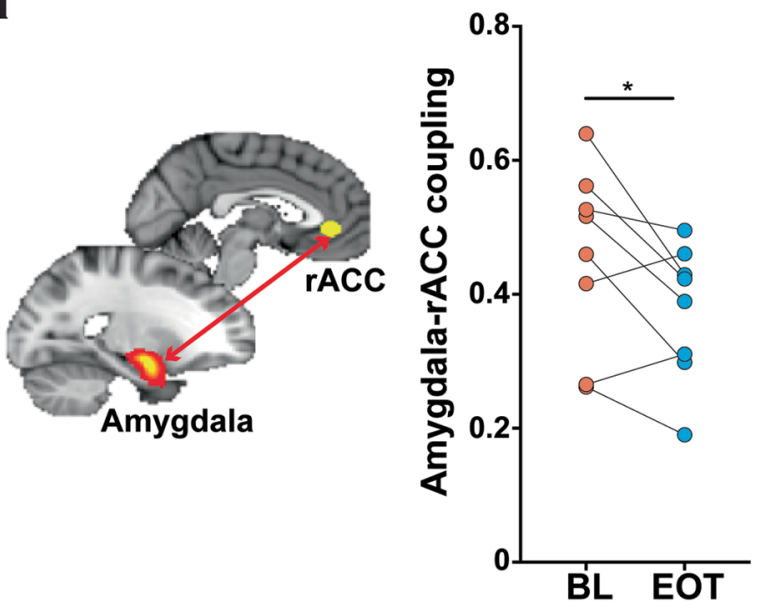

Vineland Adaptive Behavior Scales (VABS) Scores
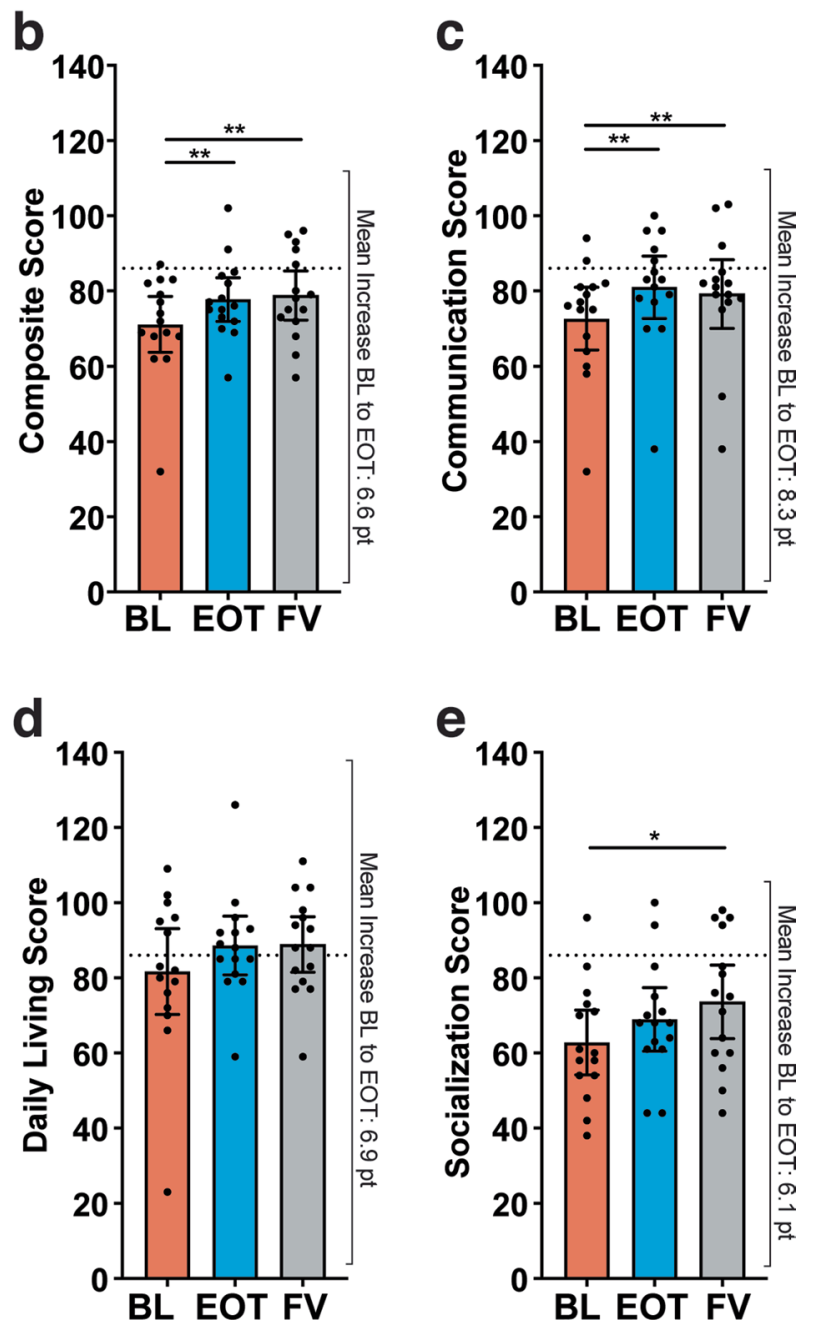

Extended Data Fig. 4 | See next page for caption. 
Extended Data Fig. 4 | Altered amygdalar functional connectivity and Vineland Adaptive Behavior Scales (VABS) diagnostic scores. a, Functional connectivity between the amygdala and $\mathrm{rACC}$ brain regions, assessed by $\mathrm{fMRI}$ scans at BL and EOT. VABS test scores at baseline (BL, orange), the end of treatment (EOT, blue), and final visit (FV, grey) timepoints, where a lower score indicates increased severity. Dotted line indicates threshold ( $\leq 86)$ of scores categorized as moderately low and low $(n=8)$. b-d, Vineland Adaptive Behavior Scales (VABS). Exact $p$-value: 0.046 . b, Composite scores $(n=15)$. Exact adj. $p$-values: BL vs EOT, 0.004; BL vs FV, 0.003. c, Communication scores ( $n=15$ ). Exact adj. $p$-values: BL vs EOT, 0.001; BL vs FV, 0.007. d, Daily living scores $(n=15)$. e, Socialization scores $(n=15)$. Exact adj. $p$-value: 0.02. In each respective left panel, scores of all study participants with valid VABS are shown, with a dotted line marking the threshold of 86 for moderately low and low scoring individuals. Mean increase between BL and EOT timepoints is noted. Abbreviations: $\mathrm{rACC}$, rostral anterior cingulate cortex; BL, baseline; EOT, end of treatment, FV, final visit. Data analysis was conducted on the completers group, but any individual with over $25 \%$ estimated answers in any domain was removed. Data analysis is exploratory and post hoc in nature. Panel $a$ is a subset of the participants who agreed to $\mathrm{fMRI}$, analyzed by a paired t-test.shown, and panels b-e are displayed as mean and $95 \%$ confidence intervals with analysis performed by Linear Mixed Effects Analysis with multiple comparisons and false discovery rate correction ( ${ }^{\star} p a d j . \leq 0.05,{ }^{\star \star} p$ adj. $\leq 0.01,{ }^{\star \star \star}$ p adj. $\left.\leq 0.001\right)$. 
Social Responsiveness Scale (SRS) Scores
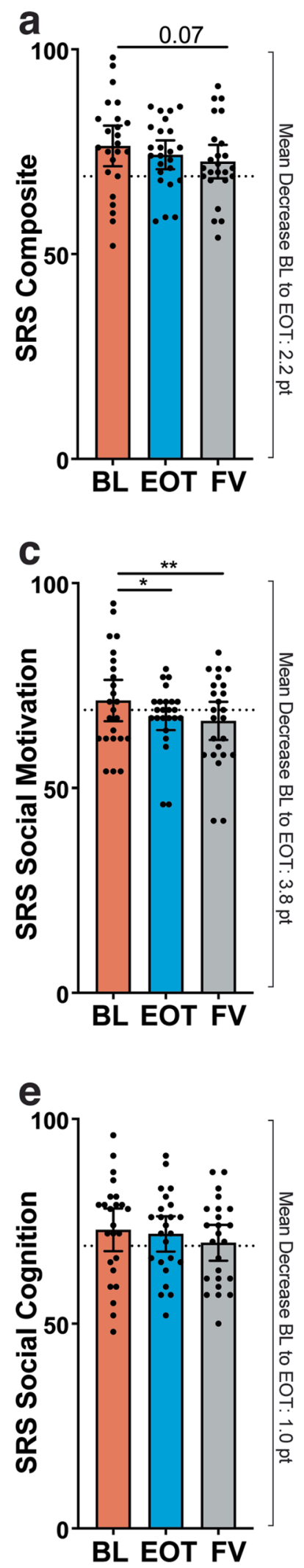
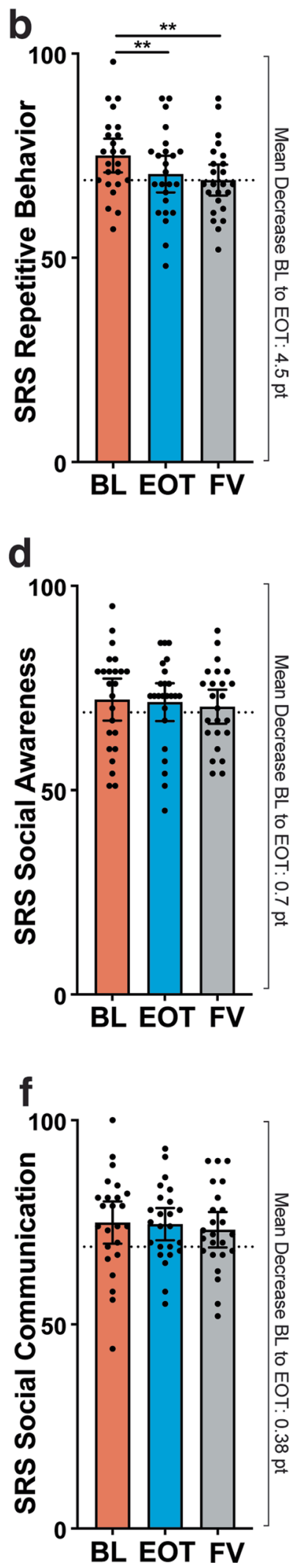

Aberrant Behavior Checklist (ABC) Scores
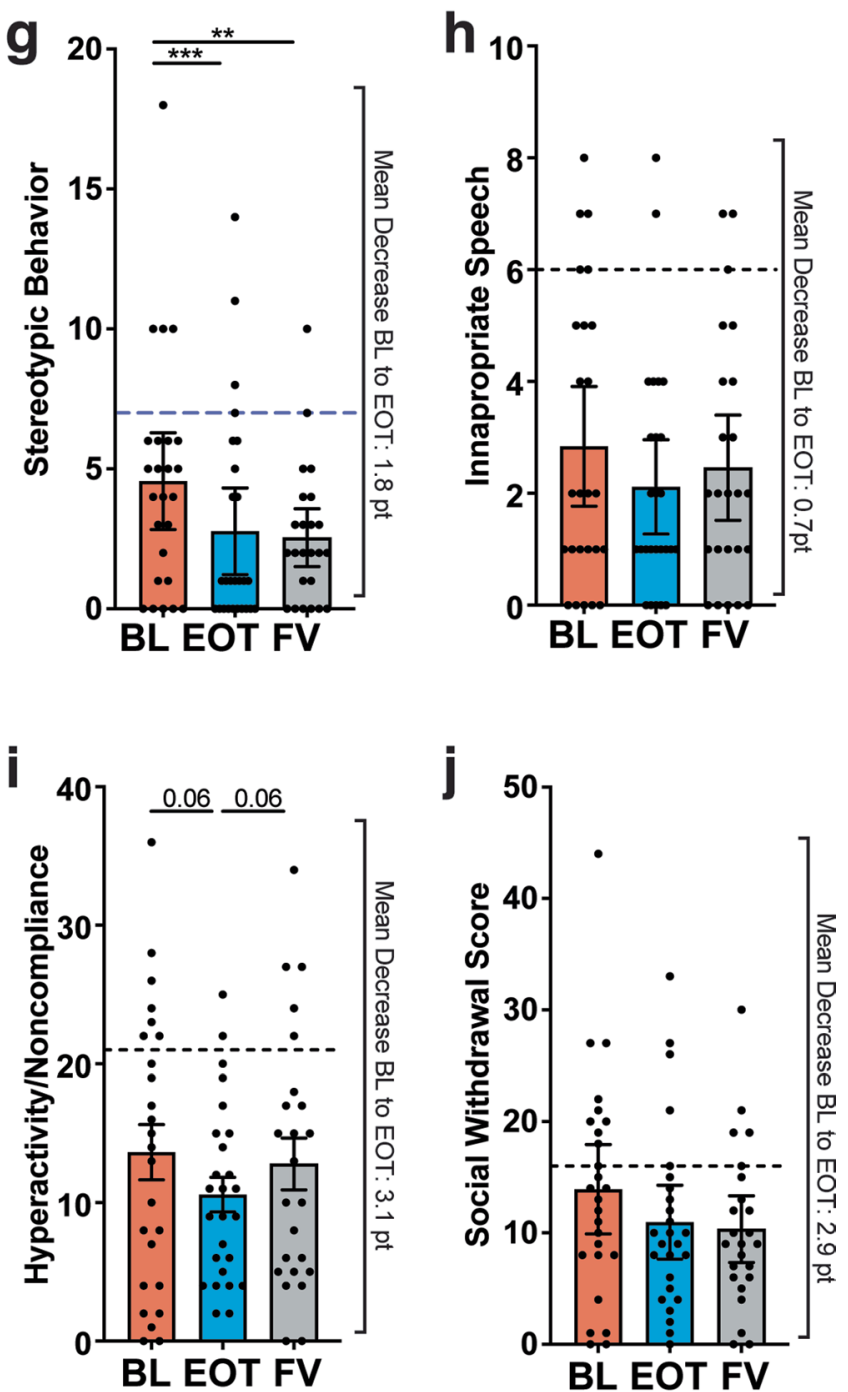

Extended Data Fig. 5 | See next page for caption. 
Extended Data Fig. 5 | Extended behavior scores of the social responsiveness scale (SRS) and Aberrant Behavior Checklist (ABC). a-f, SRS behavior scores $(n=24)$ at baseline (BL, orange), the end of treatment (EOT, blue), and final visit (FV, grey) timepoints for the composite score (a), repetitive behavior (b) (Exact adj. p-values both 0.004), social motivation (c) (Exact adj. p-values: BL vs EOT, 0.04; BL vs FV, 0.002), social awareness (d), social communication (e), and social cognition (f) domains. $\mathbf{g - j}, \mathrm{ABC}$ behavior scores at BL, EOT, and FV for all individuals for the stereotypic behavior score $(B L, E O T, n=26 ; F V, n=24)(\mathbf{g})$, inappropriate speech (Exact adj. p-values: BL vs EOT, 0.0007; BL vs FV, 0.003). (h), hyperactivity/noncompliance (i), and social withdrawal $(\mathbf{j})$ domains. Dotted lines indicate categorical thresholds for the top quartile among the ASD population. Mean increase between BL and EOT timepoints is noted. Abbreviations: BL, baseline; EOT, end of treatment; FV, final visit. Data analysis was conducted on the completers group minus two subjects who missed assessment due to parental illness. Data analysis is exploratory and post hoc in nature, shown as mean and $95 \%$ confidence intervals with statistics for performed by repeated measures one-way ANOVA with multiple comparisons and false discovery rate correction.

$\left({ }^{\star}\right.$ p adj. $\leq 0.05,{ }^{\star \star} p$ adj. $\leq 0.01,{ }^{\star \star \star} p$ adj. $\leq 0.001$ ). 


\section{Extended Data Table 1 | Exclusion criteria}

\begin{tabular}{|c|c|}
\hline Inclusion Criteria & Exclusion Criteria \\
\hline $\begin{array}{l}\text { Clinically diagnosed, documented Autism Spectrum Disorder (DSM-V criteria) confirmed with ADOS-2 at } \\
\text { Visit } 1 \text { or within } 18 \text { months prior to Visit } 1 \text {. }\end{array}$ & History of inflammatory bowel disease, bowel obstruction, diverticulosis or colon polyps. \\
\hline Adolescents $\geq 12$ and $<18$ years of age at the time of consent & Oral, injected, inhaled antibiotic within 30 days prior to Screening \\
\hline Subject and/or care giver must be able to read/write in English. & Currently taking a controlled-or extended-release medication \\
\hline $\begin{array}{l}\text { Able to provide developmentally and age-appropriate written informed consent as required per local } \\
\text { regulations for subject and/ or legal guardian. }\end{array}$ & A febrile gastrointestinal illness involving $>1$ day of diarrhea within 30 days prior to Screening \\
\hline $\begin{array}{l}\text { Male subjects who are post-pubertal must be sterile (surgically or otherwise) for at least } 6 \text { months or are } \\
\text { using single barrier contraception during the duration of the trial and up until } 1 \text { month after the last dose } \\
\text { AB-2004. } \\
\text { OR } \\
\text { Female subjects that are not lactating and have a negative pregnancy test at the Screening and who are } \\
\text { surgically sterile for at least } 6 \text { months or who agree to use double-barrier contraception, an } \\
\text { intrauterine device, or an oral contraceptive over the duration of the trial and at least } 4 \text { weeks after the } \\
\text { last dose of AB-2004 }\end{array}$ & History of significant gastric or intestinal surgery (excluding appendectomy). \\
\hline Subjects who weigh a minimum of the $5^{\text {th }}$ - percentile for their age at Screening & History of diabetes. \\
\hline $\begin{array}{l}\text { On a stable therapeutic regimen-including medication, supplements, and probiotics-for at least } 30 \\
\text { days prior to Screening and while participating in the trial. }\end{array}$ & $\begin{array}{l}\text { Have any clinically significant condition, is immunocompromised, or has an unstable intercurrent illness } \\
\text { that would, in the opinion of the Investigator, preclude study participation or interfere with safety and } \\
\text { tolerability assessments of the subject. }\end{array}$ \\
\hline $\begin{array}{l}\text { Good health, as determined by the Principal Investigator }(P \mathrm{P}) \text { or qualified designee, on the basis of } \\
\text { medical history, physical examination and screening laboratory results. }\end{array}$ & Have an active malignancy or history of malignancy within 2 years prior to Screening. \\
\hline $\begin{array}{l}\text { Good health, as determined by the Principal Investigator }(\mathrm{Pl}) \text { or qualified designee, on the basis of } \\
\text { medical history, physical examination and screening laboratory results. }\end{array}$ & History of significant head injury within 6 months prior to Screening. \\
\hline \multirow[t]{9}{*}{$\begin{array}{l}\text { History of gastrointestinal symptoms (diarrhea, constipation, abdominal pain, bloating) confirmed in the } e \\
\text { diary with at least } 50 \% \text { compliance of entry for at least } 14 \text { days during the screening period. }\end{array}$} & Clinically significant blood pressure abnomalities. \\
\hline & $\begin{array}{l}\text { Have a comorbid major psychiatric condition (e.g., schizophrenia or bipolar disorder) at Screening that } \\
\text { in the opinion of the Investigator may interfere with the subject's ability to complete study } \\
\text { procedures/comply with study requirements. }\end{array}$ \\
\hline & $\begin{array}{l}\text { Have met criteria for a moderate to severe substance use disorder as described within the } \\
\text { Diagnostic and Statistical Manual of Mental Disorders } V(D S M-5) \text { criteria within } 5 \text { years prior to } \\
\text { Screening. }\end{array}$ \\
\hline & $\begin{array}{l}\text { Have a history of positive hepatitis B (surface antigen) and/or hepatitis } C \text { virus antibody lab results or } \\
\text { acquired immunodeficiency syndrome (AIDS). }\end{array}$ \\
\hline & $\begin{array}{l}\text { Have a positive urine toxicology screen for drugs of abuse (non-prescribed amphetamines, } \\
\text { benzodiazepines, barbiturates, cannabinoids, or cocaine). }\end{array}$ \\
\hline & Have a positive alcohol test. \\
\hline & In the investigator's opinion at significant risk for suicidal behavior \\
\hline & $\begin{array}{l}\text { Have received any investigational product or device or participated in another investigational study } \\
\text { within } 30 \text { days before the Screening visit; or received an investigational live bacterial product or fecal } \\
\text { matter transplant within } 90 \text { days before the Screening visit; or is scheduled to receive an investigational } \\
\text { device or another investigational drug during the course of this study. }\end{array}$ \\
\hline & $\begin{array}{l}\text { Are from vulnerable populations, including but not limited to employees (temporary, part-time, full-time, } \\
\text { etc), or a family member of the research staff conducting the study, or of the Sponsor, or the Clinical } \\
\text { Research Organization or of the Institutional Review Board. }\end{array}$ \\
\hline
\end{tabular}




\section{Extended Data Table 2 | Schedule of assessments}

\begin{tabular}{|c|c|c|c|c|c|c|c|c|c|c|c|c|c|c|c|c|}
\hline & & & \multirow{2}{*}{\begin{tabular}{|c|}
$\begin{array}{c}\text { Screening } \\
\text { Period }\end{array}$ \\
$\begin{array}{c}\text { Screening } \\
\text { Visit }^{\mathrm{k}}\end{array}$ \\
\end{tabular}} & \multicolumn{12}{|c|}{ Treatment Period } & \multirow{2}{*}{$\begin{array}{c}\text { Recovery Period } \\
\text { Follow-up }\end{array}$} \\
\hline & & & & \multicolumn{2}{|c|}{ Dose Period 1 Start } & \begin{tabular}{|c|} 
Telephone \\
Safety \\
Check
\end{tabular} & \multicolumn{2}{|c|}{ Dose Period 2 Start } & \multicolumn{2}{|c|}{$\begin{array}{l}\text { Telephone Safety } \\
\text { Check }\end{array}$} & \multicolumn{2}{|c|}{ Dose Period 3 Start } & \multicolumn{2}{|c|}{$\begin{array}{l}\text { Telephone Safety } \\
\text { Check }\end{array}$} & EOT & \\
\hline \multicolumn{3}{|c|}{ Visit } & V1 (BL) & \multicolumn{2}{|c|}{ V2 } & & \multicolumn{2}{|c|}{ V3 } & & & \multicolumn{2}{|c|}{ V4 } & & \begin{tabular}{|l|} 
V5 (EOT) \\
\end{tabular} & V6 (FV) \\
\hline \multirow{2}{*}{\multicolumn{3}{|c|}{$\begin{array}{c}\text { AB-2004 Trial Day } \\
\text { PROCEDURES }\end{array}$}} & -28 to -1 & \multirow{2}{*}{\multicolumn{2}{|c|}{$\frac{1}{\text { Day } 1}$}} & $8(+2)$ & \multirow{2}{*}{\multicolumn{2}{|c|}{$\frac{15(+5)}{\text { Week } 2}$}} & \multicolumn{2}{|c|}{$22(+2)$} & \multirow{2}{*}{\multicolumn{2}{|c|}{$\begin{array}{l}29(+5) \\
\text { Week } 4\end{array}$}} & & & \begin{tabular}{|l|}
$57(+5)$ \\
\end{tabular} & $85( \pm 5)$ \\
\hline & & & & & & & & & & & & & \multicolumn{2}{|c|}{$43(+2)$} & Week 8 & Week 12 \\
\hline \multicolumn{3}{|c|}{ Informed Consent/Assent } & $x$ & & & & & & & & & & & & & \\
\hline Demographic Data & & & $x$ & & & & & & & & & & & & & \\
\hline \begin{tabular}{|l|} 
Vital Signs ${ }^{\mathrm{a}}$ \\
\end{tabular} & & & $\mathrm{x}$ & $\underline{x}$ & K & & $x$ & & & & $\bar{x}$ & & & & $\bar{x}$ & $\bar{x}$ \\
\hline $\begin{array}{l}\text { Medical/Surgical, G } \\
\text { diet), 6-GSI }\end{array}$ & History (including & & $x$ & & & & & & & & & & & & & \\
\hline Prior \& Concomitan & t Meds & & $x$ & $\underline{x}$ & r & $\mathrm{x}$ & $\underline{x}$ & & & & $\underline{x}$ & & 2 & $x$ & $\mathrm{X}$ & $x$ \\
\hline & & Full & $\bar{x}$ & & & & & & & & & & & & $\bar{x}$ & $\bar{x}$ \\
\hline Physical Examb & & Abbreviated & & $x$ & r & & $x$ & & & & $x$ & & & & & \\
\hline Inclusion/Exclusion & Criteria & & $x$ & $\bar{x}$ & & & $\bar{x}$ & & & & $\bar{x}$ & & & & & \\
\hline \begin{tabular}{|l} 
Adverse Events \\
\end{tabular} & & & $x$ & $\underline{x}$ & & $x$ & $\underline{x}$ & & & & $\underline{x}$ & & $\underline{x}$ & $\bar{x}$ & $x$ & $x$ \\
\hline Set up download \& & Train on & \begin{tabular}{|l} 
Survey \\
training
\end{tabular} & $x$ & & & & & & & & & & & & & \\
\hline e-diary ${ }^{\circ}$ & & \begin{tabular}{|l|} 
Drug diary \\
training
\end{tabular} & & $x$ & & & & & & & & & & & & \\
\hline $\begin{array}{l}\text { Review of e-diary/pe } \\
\text { compliance }\end{array}$ & aper diary & & $x$ & $x$ & & $x$ & $x$ & & & & $x$ & & & $x$ & $x$ & $x$ \\
\hline & & \begin{tabular}{|l|}
$\begin{array}{l}\text { Volume \& } \\
\text { pH }\end{array}$ \\
\end{tabular} & $x$ & $x$ & & & 0 & & & & $x$ & & & & $x$ & $x$ \\
\hline Home Collection - U & Jrine \& Stool ${ }^{d}$ & $\begin{array}{l}\text { Urine } \\
\text { Biomarkers }\end{array}$ & $x$ & $x$ & & & 0 & & & & $x$ & & & & $x$ & $x$ \\
\hline & & $\begin{array}{l}\text { Stool } \\
\text { Biomarkers }\end{array}$ & $x$ & $x$ & & & 0 & & & & $x$ & & & & $x$ & $x$ \\
\hline & & & Screenin & g Period & & & & & & atment Per & & & & & & Recovery Period \\
\hline $\begin{array}{l}\text { Table } \\
\text { (Conti }\end{array}$ & $\begin{array}{l}\text { 3: Schedule of Ass } \\
\text { inued) }\end{array}$ & essments & Screenin & ing Visitk & \begin{tabular}{|c|} 
Dose \\
Period 1 \\
Start \\
\end{tabular} & \begin{tabular}{|c|} 
Telephone \\
Safety \\
Check
\end{tabular} & $\begin{array}{c}\text { Dose } \\
\text { Period 2 } \\
\text { Start } \\
\end{array}$ & $\begin{array}{l}\text { Telephon } \\
\text { Che }\end{array}$ & $\begin{array}{l}\text { e Safety } \\
\text { ck }\end{array}$ & Dose Per & iod 3 Start & $\begin{array}{l}\text { Telephon } \\
\text { Che }\end{array}$ & $\begin{array}{l}\text { e Safety } \\
\text { ck }\end{array}$ & & OT & Follow-up \\
\hline & Visit & & $\mathbf{v}$ & 11 & V2 & & V3 & & & & 14 & & & & $\sqrt{5}$ & V6 \\
\hline & B-2004 Trial Day & & -28 & --1 & 1 & $8(+2)$ & $15(+5)$ & $22(-7)+1)$ & & & $(+5)$ & 43( & & & $(+5)$ & $85( \pm 5)$ \\
\hline & PROCEDURES & & & & Day 1 & & Week 2 & & & & ek 4 & & & & ek 8 & Week 12 \\
\hline & Hematology \& Seru & m Chemistry & $\mathrm{x}$ & $x$ & 0 & & 0 & & & & $x$ & & & & $x$ & 0 \\
\hline Blood Labs' & Blood Biomarkers $^{\circ}$ & & $\bar{x}$ & $\bar{x}$ & 0 & & $\bar{O}$ & & & & $\bar{x}$ & & & & $\bar{x}$ & $\bar{O}$ \\
\hline & Genetic Sample & & & & 0 & & & & & & & & & & & \\
\hline Hring t he & Urinalysis & & $\mathrm{x}$ & $x$ & 0 & & $\mathrm{O}$ & & & & $x$ & & & & $\mathrm{x}$ & 0 \\
\hline Uine Lads & Drug \& Alcohol ${ }^{9}$ & & $x$ & $x$ & $\mathrm{O}$ & & o & & & & $x$ & & & & $x$ & $\mathrm{O}$ \\
\hline Pregnancy Test ${ }^{h}$ & & & $\mathrm{x}$ & $x$ & $x$ & & o & & & & $x$ & & & & $x$ & O \\
\hline $\begin{array}{l}\text { Lactulose/Mannitol } \\
\text { Test }\end{array}$ & & & $x$ & $x$ & & & & & & & & & & & $x$ & \\
\hline & ADOS-2 (if required & & $\bar{x}$ & & & & & & & & & & & & & \\
\hline & CGI-S & & $\bar{x}$ & & $x$ & & & & & & $\bar{x}$ & & & & $\bar{x}$ & $x$ \\
\hline Clinician & CGI-I & & & & & & & & & & $\bar{x}$ & & & & $\bar{x}$ & $\bar{x}$ \\
\hline & PARS & & $\bar{x}$ & & & & & & & & & & & & $\bar{x}$ & $\bar{x}$ \\
\hline & Vinelands 3 & & $\bar{x}$ & & & & & & & & & & & & $\bar{x}$ & $\bar{x}$ \\
\hline & GSRS, Dietary Que & stionnaire & $\bar{x}$ & $\mathrm{~K}$ & $x$ & & $\mathrm{X}$ & & & & $\bar{x}$ & & & & $\bar{x}$ & $x$ \\
\hline Patient & $\begin{array}{l}\text { ABC, RBS-R, } \\
\text { SRS-2, CASI-5 } \\
\end{array}$ & & $\mathrm{x}$ & & & & & & & & & & & & $x$ & $x$ \\
\hline Questionnaires & \begin{tabular}{|l}
$\begin{array}{l}\text { BSS \& NRS } \\
\text { (daily e-diary) }\end{array}$ \\
\end{tabular} & & $x$ & & $x$ & $x$ & $x$ & $x$ & & & $x$ & $x$ & & & $x$ & $x$ \\
\hline $\begin{array}{l}\text { Dispense /Collect } \\
\text { Study Medication }\end{array}$ & & & & & $x$ & & $x$ & & & & $x$ & & & & $x$ & \\
\hline MRIScan' & & & & & 0 & & & & & & & & & & 0 & \\
\hline
\end{tabular}

Rey. X: Mandatory, O: Optional.

a) Vital signs included body weight, pulse rate (beats per minute), blood pressure (systolic and diastolic blood pressure, $\mathrm{mm} \mathrm{Hg}$ ), and respiratory rate (respirations per minute) after at least 2 minutes at rest in a supine position. b) Physical Examination (PE) - full PE including at least general appearance, head (eyes, ears, nose, mouth, throat), skin, neurological, musculoskeletal, cardiovascular, respiratory, abdomen and extremities; abbreviated PE was c) An e-diary was used to assess Gl symptoms, including pain, additional electronic patient reported outcomes (BSS and NRS) and IP dosing. A paper diary was provided to subjects to use if there were technical issues with thed) Home collection samples. First sample was to be collected within 1 week of Visit 1 and subsequent samples were to be collected 2 to 3 days before the next scheduled visit.

e) Biomarker assessments performed in samples from blood (plasma or serum), urine, and feces. Blood: intestinal permeability (eg, zonulin, fatty acid-binding protein 2, $a-1$ antitypsin, lipopolysaccharide binding protein) and f) Laboratory tests to establish eligibility were to be completed within $\mathbf{2 8}$ days prior to enrollment and results reviewed by the Investigator or authorized delegate before enrollment

g) Drug of Abuse Screen - dipstick urine drug tests were screened for non-prescribed cannabis, cocaine, amphetamines, benzodiazepines, and barbiturates to ensure subjects were not self-medicating during the study. A separate h) Pregnancy testing was applicable to female subjects only. The test was to be conducted on either a urine or serum sample.

i) BSS and NRS were captured in the e-diary/paper diary on a daily basis.

j) MRIs were performed on a subset of subjects $(\sim 10)$. All subjects were to be offered an MRI at sites where MRIs were available.

k) Screening visit was to be conducted over 2 to 3 visits. Consent/assent, assessments to confirm eligibility, and initiation of the e-diary/paper diary were to be completed at the first part of Visit 1 , whereas other tests were to be

Abbreviations: 4-EPS = 4-Ethylphenyl Sulfate; 5HIAA = 5-Hydroxyindoleacetic acid; 6-GSI = Gastrointestinal Severity Index; ABC = Aberrant Behavior Checklist; ADOS-2 = Autism Diagnostic Observational Schedule, Second Edition; BSS = Bristol Stool Scale; CASI-5 = Child and Adolescent Symptom Inventory 5; CGI-I = Clinical Global Impression-Improvement; CGI-S = Clinical Global Impression-Severity; EOT = End of Treatment; GI = gastrointestinal; GSRS =

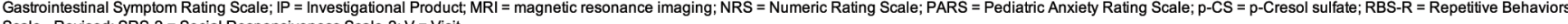
Scale - Revised; SRS-2 = Social Responsiveness Scale-2; $V$ = Visit. 


\section{NATURE MEDICINE}

Extended Data Table 3 | Metabolite and behavior correlations

\begin{tabular}{|c|c|c|c|c|c|c|c|c|c|}
\hline \multirow[b]{2}{*}{ Metabolite } & \multirow[b]{2}{*}{ Sample } & \multicolumn{2}{|c|}{$\begin{array}{l}\text { High BL PARS } \\
\qquad(n=15)\end{array}$} & \multicolumn{2}{|c|}{$\begin{array}{c}\text { All subjects PARS } \\
\qquad(n=26)\end{array}$} & \multicolumn{2}{|c|}{$\begin{array}{l}\text { High BL ABC-I } \\
\qquad(n=11)\end{array}$} & \multicolumn{2}{|c|}{$\begin{array}{l}\text { All subjects ABC-I } \\
\qquad(n=26)\end{array}$} \\
\hline & & $r$ & p-Value & $\mathbf{r}$ & p-Value & $\mathbf{r}$ & p-Value & $\mathbf{r}$ & p-Value \\
\hline $3-1 S$ & Plasma & -0.11 & 0.70 & 0.20 & 0.35 & 0.03 & 0.89 & 0.03 & 0.89 \\
\hline 4-EPS & Plasma & 0.35 & 0.22 & -0.16 & 0.45 & -0.03 & 0.90 & -0.03 & 0.90 \\
\hline $\mathrm{HHA}$ & Plasma & 0.39 & 0.17 & -0.18 & 0.42 & 0.07 & 0.75 & 0.07 & 0.75 \\
\hline HPAA & Plasmá & -0.47 & 0.09 & -0.52 & 0.01 & -0.32 & 0.12 & -0.32 & 0.12 \\
\hline HPHPA & Plasma & 0.37 & 0.20 & -0.27 & 0.22 & 0.04 & 0.86 & 0.04 & 0.86 \\
\hline HPPA & Plasmá & 0.42 & 0.13 & 0.29 & 0.18 & 0.02 & 0.92 & 0.02 & 0.92 \\
\hline$p-C G$ & Plasma & 0.08 & 0.79 & 0.18 & 0.41 & 0.10 & 0.64 & 0.10 & 0.64 \\
\hline$p-C S$ & Plasma & 0.00 & 0.99 & 0.31 & 0.15 & 0.05 & 0.79 & 0.05 & 0.79 \\
\hline $3-I S$ & Urine & 0.32 & 0.24 & -0.01 & 0.98 & 0.02 & 0.93 & 0.02 & 0.93 \\
\hline 4-EPS & Urine & 0.28 & 0.31 & -0.34 & 0.12 & -0.18 & 0.39 & -0.18 & 0.39 \\
\hline $\mathrm{HHA}$ & Urine & 0.00 & 0.99 & -0.23 & 0.28 & -0.23 & 0.27 & -0.23 & 0.27 \\
\hline HPAA & Urine & -0.37 & 0.17 & -0.39 & 0.06 & -0.36 & 0.07 & -0.36 & 0.07 \\
\hline HPHPA & Urine & -0.04 & 0.88 & -0.25 & 0.26 & -0.25 & 0.23 & -0.25 & 0.23 \\
\hline HPPA & Unine & -0.18 & 0.51 & 0.00 & 0.98 & -0.31 & 0.13 & -0.31 & 0.13 \\
\hline$p-C G$ & Urine & 0.13 & 0.63 & 0.22 & 0.31 & -0.05 & 0.82 & -0.05 & 0.82 \\
\hline$p-C S$ & Urine & 0.21 & 0.45 & 0.09 & 0.67 & -0.17 & 0.42 & -0.17 & 0.42 \\
\hline
\end{tabular}

Two-tailed Pearson's correlations between changes in behavior scores and changes in metabolite levels, not corrected for multiple comparisons. 


\section{Reporting Summary}

Nature Portfolio wishes to improve the reproducibility of the work that we publish. This form provides structure for consistency and transparency in reporting. For further information on Nature Portfolio policies, see our Editorial Policies and the Editorial Policy Checklist.

\section{Statistics}

For all statistical analyses, confirm that the following items are present in the figure legend, table legend, main text, or Methods section.

$\mathrm{n} / \mathrm{a} \mid$ Confirmed

$\bigotimes$ The exact sample size $(n)$ for each experimental group/condition, given as a discrete number and unit of measurement

\ A statement on whether measurements were taken from distinct samples or whether the same sample was measured repeatedly

The statistical test(s) used AND whether they are one- or two-sided

Only common tests should be described solely by name; describe more complex techniques in the Methods section.

\ A description of all covariates tested

$\square$ A description of any assumptions or corrections, such as tests of normality and adjustment for multiple comparisons

A full description of the statistical parameters including central tendency (e.g. means) or other basic estimates (e.g. regression coefficient)

AND variation (e.g. standard deviation) or associated estimates of uncertainty (e.g. confidence intervals)

For null hypothesis testing, the test statistic (e.g. $F, t, r$ ) with confidence intervals, effect sizes, degrees of freedom and $P$ value noted

Give $P$ values as exact values whenever suitable.

Х $\square$ For Bayesian analysis, information on the choice of priors and Markov chain Monte Carlo settings

Х $\square$ For hierarchical and complex designs, identification of the appropriate level for tests and full reporting of outcomes

$\square \bigotimes$ Estimates of effect sizes (e.g. Cohen's $d$, Pearson's $r$ ), indicating how they were calculated

\section{Our web collection on statistics for biologists contains articles on many of the points above.}

\section{Software and code}

Policy information about availability of computer code

Data collection Ethovision v.10; Patient data was collected using IMedNet (v1.94.0); e-diary designed to collect GI data was a custom application developed by Dedo (www.dedo.ai) ; fMRI data was collected using GE Discovery 750, software level DV26.0, Siemens Skyra, software level VE11, Siemens Prisma Fit, software level VE11

Data analysis GraphPad Prism v.8, 9

SAS v.9.4

MS Excel v.2012

fMRIPrep 20.0.4

BIDS 1.2.1

FreeSurfer 6.0.1

For manuscripts utilizing custom algorithms or software that are central to the research but not yet described in published literature, software must be made available to editors and

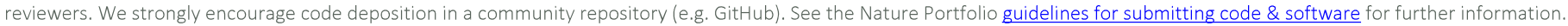


Policy information about availability of data

All manuscripts must include a data availability statement. This statement should provide the following information, where applicable:

- Accession codes, unique identifiers, or web links for publicly available datasets

- A description of any restrictions on data availability

- For clinical datasets or third party data, please ensure that the statement adheres to our policy

Axial Therapeutics ("Axial"), under certain restrictions below, will provide access to at least the minimum data sets from its clinical trials that are necessary to interpret, verify and extend the research findings. Axial considers the minimum data sets may include the study protocol (already provided as supplemental data), and the tables, lists and figures (TLFs) from the final Clinical Study Report. Such data sets will be made available only to qualified scientific researchers for legitimate scientific research purposes. Simultaneously, Axial is mandated by law and otherwise is committed to protecting the privacy and rights of all subjects who participate in its clinical trials; therefore, all data supplied will be de-identified according to applicable regulations.

Data requests should be sent to corresponding author A. Stewart Campbell at stewart@axialtx.com (Subject Line: "AB-2004 Clinical Trial Data Request"); all data requests must contain clearly described in a written proposal, including statement of research purpose, research plan and methods (including statistical analysis plan, if applicable), key research personnel and data sharing plans. All requests meeting submission requirements will promptly be considered for scientific merit by internal and external (if necessary) subject matter experts and compliance personnel, and a written response approving or denying access to data will be provided. Approved requests will result in access to the necessary data through a secure data sharing portal, and access will be granted for a pre-specified defined period of time that is commensurate with the research plan. Under approved requests, Axial and the interested party must enter a Data Sharing Agreement that will govern the terms and conditions for use, storage and communication of the data, and terms for co-authorship resulting from any publication of the research results. It is recognized that certain regulations may apply in different countries, states or regions that may impact what data may be shared and with whom; Axial cannot guarantee access to the requested data in these circumstances.

\section{Field-specific reporting}

Please select the one below that is the best fit for your research. If you are not sure, read the appropriate sections before making your selection.

Life sciences

Behavioural \& social sciences

Ecological, evolutionary \& environmental sciences

For a reference copy of the document with all sections, see nature.com/documents/nr-reporting-summary-flat.pdf

\section{Behavioural \& social sciences study design}

All studies must disclose on these points even when the disclosure is negative.

Study description

Research sample

Sampling strategy

Data collection

Timing

Data exclusions

Non-participation
The AXL-2004-001 study (ANZCTR (http://www.anzctr.org.au/) ACTRN12618001956291) was an open-label, outpatient, multiple ascending dose Phase 1b/2a study in an ASD-diagnosed adolescent (12-<18 years old) population with confirmed gastrointestinal symptoms (e.g. diarrhea, constipation, abdominal pain, bloating).

ASD-diagnosed adolescent (12 - <18 years old) population with confirmed gastrointestinal symptoms (e.g. diarrhea, constipation, abdominal pain, bloating), across three sites in Australia and New Zealand, including the Queensland Children's Hospital in Brisbane (14 subjects), Brain and Mind Centre in Sydney (6 subjects), and Optimal Clinical Trials in Auckland (10 subjects).

A sample size of 25 was selected given that this number of subjects was considered sufficient in evaluating the safety and tolerability of the product which was the primary endpoint of the study (Shen, Jie, et al. "Design and conduct considerations for first-in-human trials." Clinical and translational science 12.1 (2019): 6-19). Given the extended history of safety for this product in other populations the likelihood of safety events in this study was considered low.

Patient data was collected using IMedNet (v1.94.0); e-diary designed to collect GI data was a custom application developed by Dedo (www.dedo.ai). Participants and their caregivers were present during each clinic visit. Both participants and caregivers participated in the completion of clinical assessments as dictated by the study protocol. This was an open-label study and as such researchers were not blinded to the experimental condition or study hypothesis.

41 individuals were screened between April 18, 2019 and January 23, 2020. The last visit of the study was completed on May 15, 2020.

One subject, the female participant, was included in the Safety Population, but was not included in the exploratory efficacy analysis. This subject was removed from the exploratory efficacy analysis because their participation in the trial coincided with the initial COVID-19 pandemic outbreak and its associated societal restrictions put into effect in Australia. These restrictions prevented the subject from conducting normal routines and accessing normal services. As determined by the site Principal Investigator, these abrupt changes in routine had an impact on the behavior of the participant; therefore, this subject was excluded from the efficacy analysis.

A total of 41 adolescent subjects, aged 12-17 years inclusive, were screened for eligibility for participation in the study, and the 30 who met the study-specific eligibility criteria were enrolled and received at least one dose of AB-2004 (Safety Population). One participant withdrew after the first dose due to the investigator's decision based on the subject presenting with an unrelated viral infection. Another subject withdrew consent during the low dose period due to anticipated admission to hospital for pre-existing behavioral difficulties. One participant withdrew due to significant study non-compliance, and two did not complete FV assessments 


\section{Reporting for specific materials, systems and methods}

We require information from authors about some types of materials, experimental systems and methods used in many studies. Here, indicate whether each material, system or method listed is relevant to your study. If you are not sure if a list item applies to your research, read the appropriate section before selecting a response.

\begin{tabular}{|c|c|c|c|}
\hline \multicolumn{2}{|r|}{ Materials \& experimental systems } & \multicolumn{2}{|c|}{ Methods } \\
\hline$n / a$ & Involved in the study & $n / a$ & Involved in the study \\
\hline$\bigotimes$ & $\square$ Antibodies & Х & $\square$ ChIP-seq \\
\hline$\bigotimes$ & Eukaryotic cell lines & Х & $\square$ Flow cytometry \\
\hline Х & $\square$ Palaeontology and archaeology & Х & $\square$ MRI-based neuroimaging \\
\hline$\square$ & $\bigotimes$ Animals and other organisms & & \\
\hline$\square$ & Х Human research participants & & \\
\hline$\square$ & $\bigotimes$ Clinical data & & \\
\hline$\bigotimes$ & $\square$ Dual use research of concern & & \\
\hline
\end{tabular}

\section{Animals and other organisms}

Policy information about studies involving animals; ARRIVE guidelines recommended for reporting animal research

Laboratory animals

Wild animals

Field-collected samples

Ethics oversight

Note that full information on the approval of the study protocol must also be provided in the manuscript.

\section{Human research participants}

Policy information about studies involving human research participants

Population characteristics

30 (29 male, 1 female) adolescents aged 12 to 17 (mean 14.2 years old) with confirmed diagnosis of Autism Spectrum Disorder (DSM-5 criteria) were enrolled in the study. Mean BMI for the study cohort was 21.2 (range 13.9 - 35.6). All participants presented with GI symptoms (including but not limited to constipation, diarrhea, flatulence, or abdominal pain) at the time of enrollment.

Recruitment

Participants were recruited through clinic patient databases, on-line social media advertisements, and fliers distributed by local patient advocacy groups. Participants and their families were provided with information regarding the study and the product prior to signing the consent/assent documentation. This recruitment strategy may have biased the study sample towards parents who have a higher motivation to seek treatment for their children as compared to the broader autism community. Given the open-label study design it is unlikely that this recruitment strategy impacted the results of the study.

Ethics oversight

The protocol was approved by Health and Disability Ethics Committees (New Zealand), Children's Health Queensland Hospital and Health Service Human Research Ethics Committee, and Bellberry Human Research Ethics Committee. Written informed consent/assent was provided by participants/parents/LAR/guardians in accordance with ethics committee requirements and prior to initiating any study related activity.

Note that full information on the approval of the study protocol must also be provided in the manuscript.

\section{Clinical data}

Policy information about clinical studies

All manuscripts should comply with the ICMJE guidelines for publication of clinical research and a completed CONSORT checklist must be included with all submissions.

Clinical trial registration

Study protocol 
Data collection

Outcomes
Data was collected using both an electronic data capture system (EDC, IMedNet) and e-diary application (Dedo, www.dedo.ai). Data generated during in-clinic visits was collected using the IMednet system. Daily GI symptom data was captured by the subject or their caregiver using the e-diary application. Data collection utilizing both systems commenced on April 18, 2019 and completed on May 15,2020

This study represented the first instance where the investigative product was used in the study population. Therefore, the primary endpoint for the study was safety and tolerability as assessed by physical exams, vital signs, clinical laboratory tests, and adverse events. 